\title{
Notre terre vue du ciel
}

Savoir territorial et traduction cartographique en Terre Indigène Trincheira Bacajá (Pará, Brésil)

Our earth from the sky: Territorial knowledge and cartographic translation in Indigenous Land Trincheira Bacajá (Pará, Brazil)

\section{Stéphanie Tselouiko}

\section{OpenEdition \\ Journals}

Édition électronique

URL : http://journals.openedition.org/ethnoecologie/6072

DOI : 10.4000/ethnoecologie.6072

ISSN : $2267-2419$

Éditeur

Laboratoire Eco-anthropologie et Ethnobiologie

\section{Référence électronique}

Stéphanie Tselouiko, « Notre terre vue du ciel », Revue d'ethnoécologie [En ligne], 17 | 2020, mis en ligne le 26 juin 2020, consulté le 03 juillet 2020. URL : http://journals.openedition.org/ethnoecologie/6072 ; DOI : https://doi.org/10.4000/ethnoecologie.6072

\section{Ce document a été généré automatiquement le 3 juillet 2020}

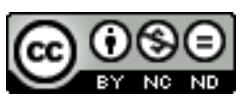

Revue d'ethnoécologie est mis à disposition selon les termes de la licence Creative Commons Attribution - Pas d'Utilisation Commerciale - Pas de Modification 4.0 International. 


\section{Notre terre vue du ciel}

Savoir territorial et traduction cartographique en Terre Indigène Trincheira Bacajá (Pará, Brésil)

Our earth from the sky: Territorial knowledge and cartographic translation in Indigenous Land Trincheira Bacajá (Pará, Brazil)

\section{Stéphanie Tselouiko}

\section{Introduction}

Situés dans la vaste région du Brésil central, dans l'État du Pará, les Xikrin, au même titre que les Kayapó (environ 12000 personnes réparties entre neuf terres indigènes), se dénomment eux-mêmes Mẽbêngôkre et appartiennent au tronc linguistique Jê². Ils occupent deux Terres Indigènes distinctes: la Terre Indigène Cateté et la Terre Indigène Trincheira Bacajá (ci-après TITB), dont il sera question dans cet article. La TITB s'insère dans un complexe multiethnique de la région actuellement désignée comme Medio Xingu, faisant face au front de colonisation agraire dans la région transamazonienne (Figure 1). D'une superficie d'environ 1500000 hectares, la TITB a été délimitée et officialisée en 1996. Les études historiques évaluent l'établissement des Xikrin aux abords de la rivière Bacajá, affluent du Xingu, entre 1924 et 1928 (Fisher 1991, 2000). 
Figure 1 : Terre Indigène Trincheira Bacajá et ses alentours en 2013

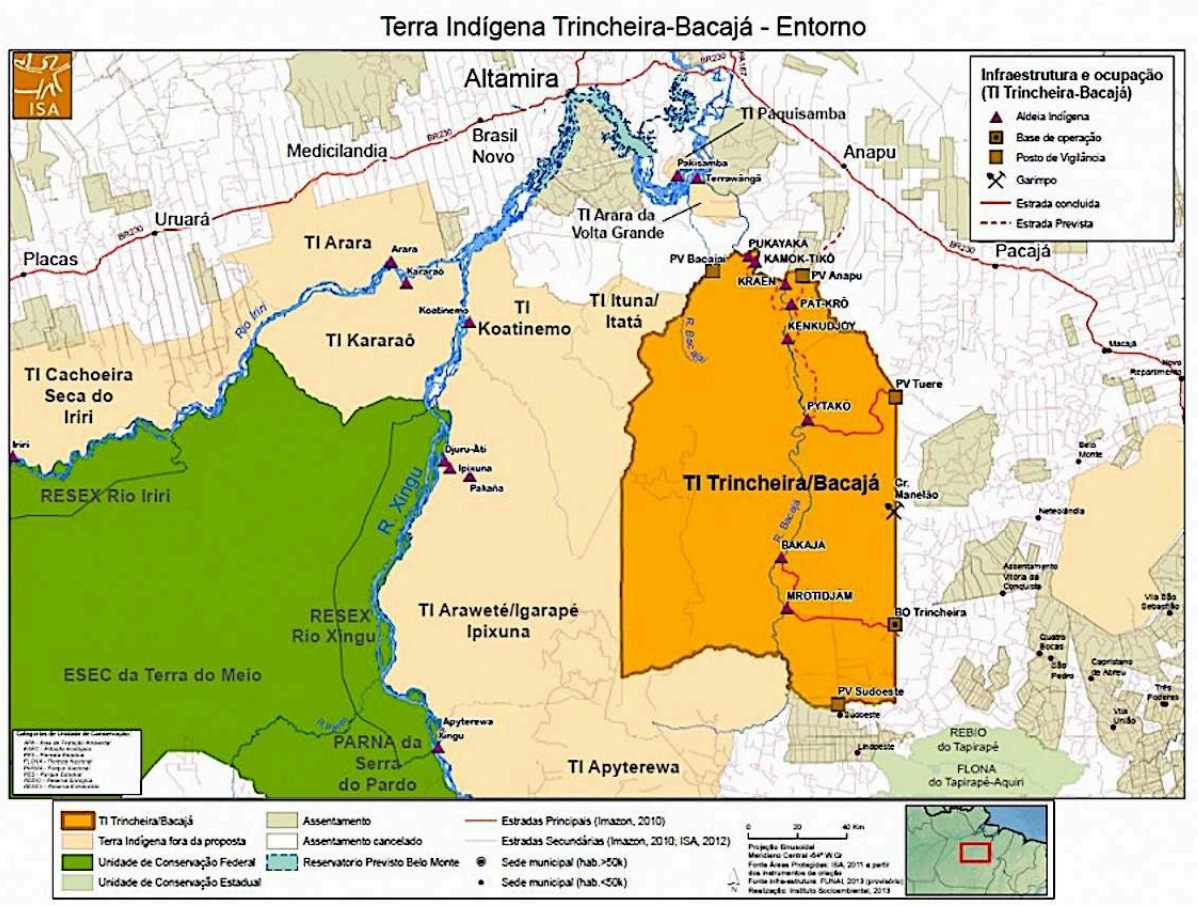

Localisation de 8 de ces 17 actuels villages, le long de la rivière Bacajá et les postes de surveillance (PV) des frontières.

Source : Instituto Socioambiental 2013

2 Chasseurs-cueilleurs semi-nomades et agriculteurs itinérants, les ancêtres des Xikrin (les Djore) ont parcouru un vaste espace du Brésil Central entre les rivières Araguaia et Xingu où ils s'établissaient préférentiellement au bord de petits cours d'eau dans la région de savane. Au cours de leurs déplacements, ils rencontrèrent de nombreux peuples avec lesquels ils entrèrent en conflit pour l'accès à la terre et aux ressources tout en maintenant des relations particulièrement hostiles avec les autres groupes Mẽbêngôkre desquels ils s'étaient séparés. Leur direction progressive vers le bassin de la rivière Bacajá, affluent du Xingu où l'un des groupes Xikrin vit actuellement, fut provoquée par les attaques des Indiens Gorotire, desquels les Djore s'étaient séparés vers le début du XIX ${ }^{e}$ siècle et par la fuite de la société nationale qui faisait de plus en plus pression dans la région. Les agents du SPI avaient tenté à plusieurs reprises de contacter les Xikrin, mais ces derniers avaient toujours résisté à ce contact qui signifiait également la pacification de leur rapport aux autres et en particulier leurs voisins, et donc, une transformation radicale de leur manière d'être, de leur condition d'existence.

3 Toutefois, affaiblis par les maladies et les guerres interethniques, les Xikrin ont finalement accepté le contact au début des années 1960, et ont été sédentarisés sur les rives de la rivière Bacajá qu'ils ont appris à connaître et à maitriser en très peu de temps se transformant en pêcheurs et navigateurs confirmés ${ }^{3}$ (Figure 2). À partir du contact "pacifique » avec la société nationale, la manière d'habiter le territoire s'est trouvée considérablement transformée. De plus, par le fait que des frontières aient été fixées définitivement, les Xikrin se sont retrouvés confinés dans un territoire (au sens législatif du terme) qui a fait émerger une nouvelle approche de la notion de la limitation (des ressources) de la terre. La limitation se trouvait être imposée par les 
frontières qui empêchaient toute projection d'expansion. Aujourd'hui, la population de la TITB est composée de plus de 1000 personnes, réparties dans dix-sept villages ${ }^{4}$.

Figure 2 : Village Bacajá en 1995

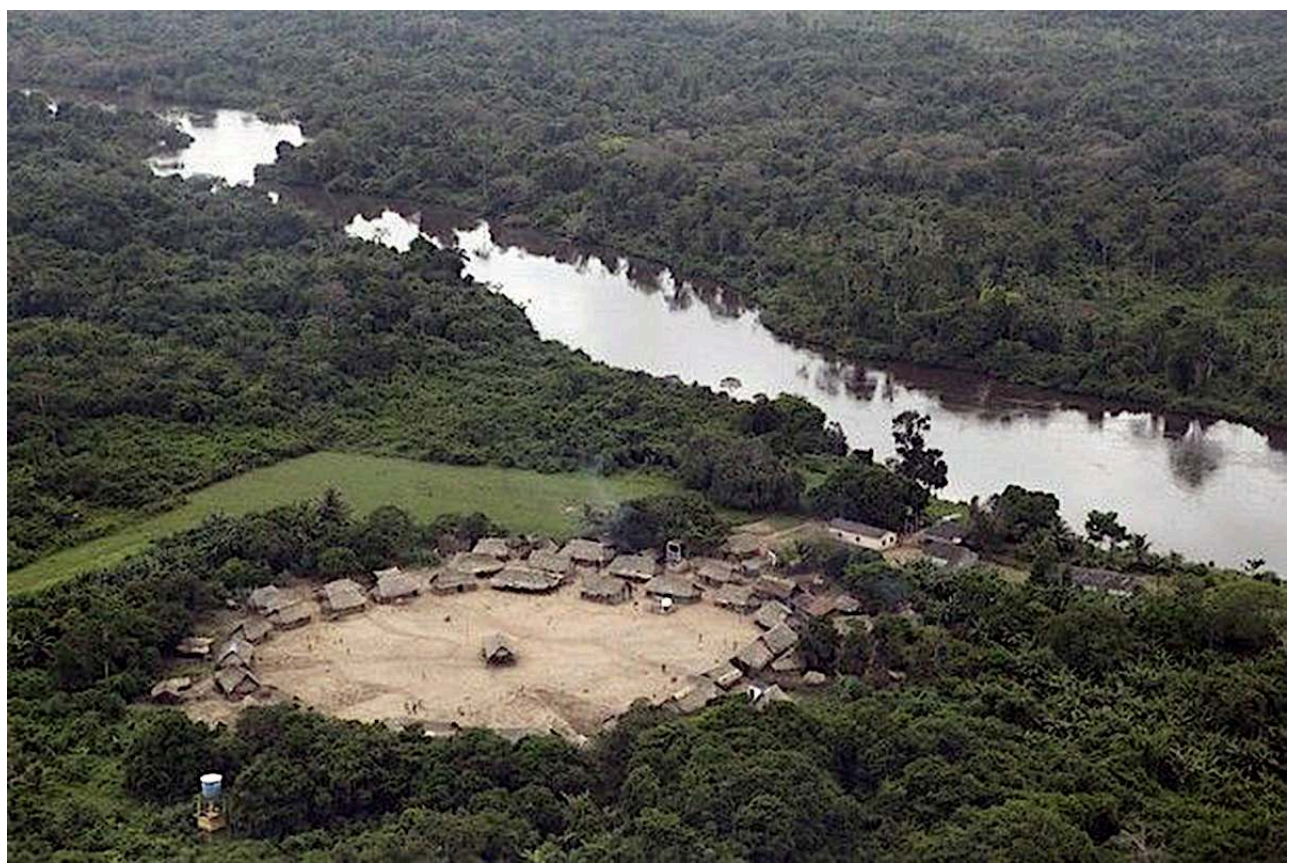

(c) C. Cohn

4 Depuis 2009, avec l'arrivée de l'usine hydroélectrique de Belo Monte (UHE Belo Monte), la mise en place d'un programme de compensation (Plano Básico Ambiental - PBA), dont la construction de routes qui relient chaque village à la Transamazonienne, la TITB est marquée par des transformations d'ordres politique, économique et écologique sans précédent et qui ne font que s'aggraver depuis avec entre autres, l'invasion par des colons de la région, des bucherons et des orpailleurs illégaux (Figure 3). 
Figure 3 : Carte des menaces réalisée par les Xikrin dans le cadre du projet d'ethnocartographie organisé par la TNC dans le cadre du programme GATI
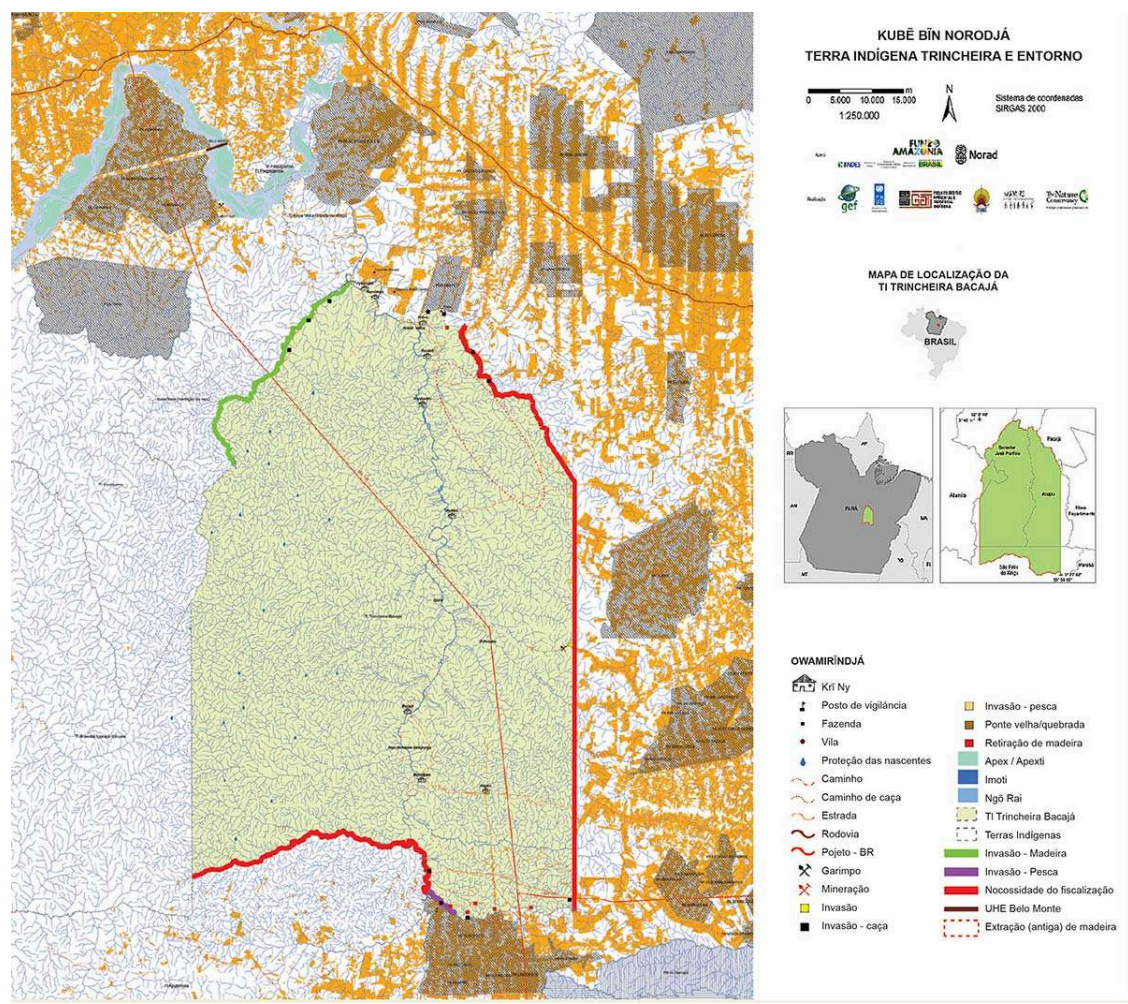

Source : Plano de Gestão Territorial e Ambiental do Povo XIKRIN da Terra Indígena Trincheira Bacajá, 2018

5 En 2013, fut initié dans tous les villages de la TITB un projet de gestion environnementale et territoriale dans le cadre d'un programme de la Politique Nationale de Gestion Environnementale et Territoriale Indigène - PNGATI (Gestão Ambiental e Territorial Indígena) sous l'égide de la Fondation Nationale de l'Indien FUNAI, avec la participation de l'ONG The Nature Conservancy - TNC. L'ethnocartographie, axe fondamental de ce projet, est présentée comme un instrument de gestion intégrée dans les terres indigènes qui

se configure dans la construction d'une carte géographique qui comprend les localités importantes de la terre indigène, leur usage culturel, la distribution spatiale des ressources naturelles, l'identification des impacts environnementaux et toutes autres informations pertinentes, garantissant l'intérêt, le regard et la compréhension indigène. L'ethnocartographie peut se faire sur la base de dessins libres, l'usage d'images satellites, des croquis, des cartes géographiques. C'est un des instruments importants dans la construction et l'établissement d'une gestion territoriale et environnementale en terre indigène. Conjointement avec le diagnostic socio-écologique, les plans de vie et autres instruments, l'ethnocartographie rend possible la construction de scénarios concernant l'usage et la conservation du territoire (PNGATI, ma traduction, mes italiques).

7 Je propose de questionner dans une approche anthropologique la mise en œuvre de ce type d'action, de plus en plus fréquent chez les populations autochtones. À partir d'une recherche ethnographique réalisée entre 2013 et 2015, cet article propose donc de mettre en évidence les mécanismes de transcription et de traduction des relations des Xikrin avec leur territoire en mots et en symboles, projetés sur des cartes. L'ambition 
d'une telle proposition est non seulement de réfléchir sur la faisabilité et les limites de l'ethnocartographie comme instrument de dialogue entre les savoirs autochtones et les connaissances scientifiques dans le diagnostic environnemental et la gestion territoriale des Terres autochtones, mais aussi de contribuer au champ de la recherche anthropologique qui se consacre à l'examen des procédures de création de documents écrits, comme les cartes et à l'émergence de nouvelles formes de gouvernance territoriales.

Dans un premier temps, j'adopterai une perspective analytique qui se concentre sur la vie quotidienne des Xikrin, en mettant en évidence leur territorialité, c'est-à-dire la relation singulière qu'ils entretiennent avec le monde forestier, dont l'habitation permanente est finalement très récente. Ce monde forestier (ou milieu) comprend aussi bien les espaces physiques (qui peuvent être désignés par le terme territoire dans son acception écologique), que les autres existants qui l'occupent. L'argument que je propose, est que territorialité et apprentissage vont de pair avec la production de la socialité qui se concrétise dans le déplacement à travers les chemins des possibles, permettant ainsi de toujours laisser ouvertes les interprétations et les improvisations dans la production d'un savoir territorial et de la pérennité de la condition Mẽbêngôkre au monde.

9 Par territorialité, j'entends une conception et une pratique singulières de l'espace vécu reflétant la manière dont un collectif donné se conçoit et entre en relation avec son milieu et les autres collectifs humains et non-humains avec qui il le partage (collectifs co-habitants) ou non (collectifs voisins, indigènes ou non). Par ailleurs, le territoire est un terme polysémique avec autant de définitions que de disciplines qui l'étudient et que de courants qui le diffusent, mais aussi que d'acteurs qui le mobilisent en fonction des contextes socio-économique et politique. Dans son sens juridique moderne, en partie influencé par l'approche éthologique de la dimension spatiale des êtres (Malmberg 1980), le territoire est souvent réduit à la clôture d'un espace soumis au contrôle d'un individu ou d'un groupe pour le défendre et optimiser l'accès aux ressources qu'il comporte, afin d'assurer la survie et la reproduction de l'individu ou du groupe en question. Dans son acception sociale, le territoire est aussi la traduction spatiale du concept de culture (Bonnemaison 1981), au fondement des notions d'identité collective ou d'ethnicité. Ces approches confondues du territoire soustendent les revendications de droits territoriaux par et pour les peuples autochtones puisque le territoire (sous-entendu ancestral) est désigné comme la condition sine qua none à leur survie.

Or, c'est justement dans cette perspective que les projets de gestion territoriale et environnementale mobilisent le terme territoire. C'est pourquoi, dans un deuxième temps, je m'attacherai à montrer comment un savoir territorial, éprouvé par le corps se transforme en nouveau savoir cartographique et comment sur la base de cette transformation, les processus relationnel et affectif qui définissent la territorialité, donnent-ils lieu à une nouvelle conception du territoire, en tant qu'objet politique.

11 Afin de saisir ce qu'est la forêt pour les Xikrin, partons sur les sentiers avec eux.

\section{«Aller en forêt »}

12 Ce qui attira mon attention lors de mes séjours en Terre Indigène Trincheira Bacajá, c'est que quelque soit l'endroit où ils étaient, les Xikrin disaient toujours «aller en 
forêt ». C'est une formule qui paraissait pourtant insensée quand elle était prononcée dans un lieu qui déjà semblait être la forêt. Certes, du point de vue de la ville, les villages des Xikrin se trouvent bien dans une zone forestière. C'est non seulement ainsi que les régionaux conçoivent l'habitat dos Indios, en opposition à la ville, mais c'est aussi la façon dont les Xikrin eux-mêmes se situent, en particulier lorsqu'ils revendiquent leur rapport singulier à ce milieu ${ }^{5}$, aujourd'hui plus que jamais menacé par les invasions provenant du front de colonisation agraire et les grands projets de développement. Mais lorsque les femmes se rencontrent chez l'une d'elles et que les hommes se réunissent au centre du village, pour décider, les uns comme les autres, des activités de chasse ou de cueillette, ils disent qu'ils vont "partir en forêt " (bàkamtẽ). Les jours qui précèdent une grande fête (metoro), les hommes disent qu'ils " partent en forêt (chasser) pour la fête» (gwajbametorobàkamtê), ce genre d'expédition collective peut se dire également mẽõt o mõrõ (= dormir en bougeant, ce qui dénote l'extension du temps d'excursion). Monter un campement se dit mêbanhôt o wadjà (mếba = nous inclusif / nhõt = forme longue du verbe dormir / wadjà en entrant : c'est-à-dire qu'on ne fait pas qu'y passer) ou encore mẽõt o wadjà = camper = dormir interné (dans la forêt). Mais le plus surprenant, c'est qu'au campement, lorsque les hommes se réunissent et décident du chemin qu'ils vont emprunter pour chasser, au moment de partir ils disent... « aller en forêt " (bàkamtê), encore. Comme si "aller en forêt » était un but inatteignable, inépuisable, une quête. Alors que signifie « aller en forêt » pour les Xikrin?

Comme nous venons de l'entreprendre, un passage par la linguistique peut nous éclairer ${ }^{6}$. Ce qui est étonnant dans cette construction linguistique bàkamtẽ, c'est que kam (qui est une position statique) et tẽ (qui signifie le mouvement et qui a besoin d'une direction, en l'occurrence ici bà, la forêt) soient juxtaposés dans la même proposition. Si bàmãwadjà se dit bien, en revanche, bàmãtẽ ne s'entend jamais, où mãtẽ signifie « aller dans une direction ». On ne va pas en forêt vers une destination précise, comme on va "sur l'eau » (ngômãtê) pour se déplacer, on va dans la forêt qui nous entoure déjà. Que ce soit en disant bàkamtẽ = « aller dans la forêt » ou bàmãwadjà = "aller à l'intérieur de la forêt ", ces deux expressions codifient soit dans le verbe, soit dans la post-position, l'idée d'être à l'intérieur de quelque chose. Kam peut aussi se trouver dans des expressions qui parlent d'activités dans un lieu. Ainsi bàkamtẽ signifierait plutôt qu'on va en forêt pour faire quelque chose en particulier, comme chasser (mrybî) ou casser des noix de palmier babaçu (ronkukwàrà). Ainsi, la forêt ne semble pas être traitée sans qu'au moment où on en parle, on pense à être à l'intérieur d'elle. Plus qu'une destination, elle est pensée comme un contexte d'action.

Cette construction linguistique qui suppose la projection de l'énonciateur dans l'espace dont il parle et dans l'action qu'il entend accomplir, permettrait à la parole d'avoir une "efficacité symbolique». Cette efficacité se voit confirmée lorsqu'au retour d'une expédition de chasse, par exemple, les hommes font le récit de leurs trajectoires et de leurs rencontres avec d'autres êtres desquelles ils sont revenus victorieux, tandis que les femmes n'auront pas besoin de parler, elles donneront à voir, simplement, leurs paniers remplis des produits de leur collecte, voire même de tortues qu'elles capturent également ${ }^{7}$. Dire «bàkamtẽ » c'est plus qu'énoncer une intention, c'est déjà affirmer que l'on reviendra avec quelque chose en plus, des fruits, du gibier, des connaissances. Dire "bàkamtẽ», c'est déjà être dans l'action. Aller en forêt sans revenir chargé est vécu comme de la malchance. Un homme dira qu'il n'y avait pas d'animal (mrykêt), de sorte qu'il ne remet pas en cause ses compétences en s'exposant à la honte (pia'am), alors que 
ce retour infortuné sera plutôt perçu par le groupe comme une faiblesse (rerekre), un manque d'habilité et de connaissance (markêt où mar signifie entendre et kêt sa négation, littéralement « ne pas entendre » qui signifie« ne pas savoir »). Ainsi, dire que l'on va en forêt c'est, en quelque sorte, déjà s'y engager avec l'expectative d'en revenir valorisé. Par quels processus se fait donc la valorisation d'une personne qui va en forêt ? L'appropriation et l'apprentissage sont les deux processus que nous aborderons dans les prochaines parties. Mais avant cela, commençons par la description des différents milieux qui composent l'espace forestier de la Terre Indigène Trincheira Bacajá, afin de saisir les conditions dans lesquelles les Xikrin évoluent et produisent un savoir territorial d'un point de vue empirique et phénoménologique.

\section{La Terre Indigène Trincheira Bacajá}

15 La TITB contient très peu de reliefs. Elle est traversée du sud au nord par la rivière Bacajá, affluent de la rivière Xingu. Le cours de la rivière est accentué par des cascades et des courants, son lit est parsemé de nombreuses pierres, ce qui demande aux navigateurs une connaissance aiguisée de son parcours pour éviter les accidents. La partie du territoire qui s'étend au-delà de la rive droite est la partie peuplée par les Xikrin. C'est celle qui fait face au front de colonisation agraire qui part de la Transamazonienne. À l'ouest, au-delà de la rive gauche, une zone importante sépare les Xikrin de leurs voisins Parakanã et Arawete contre lesquels ils furent en guerre jusqu'au contact avec la société nationale qui a imposé la pacification des relations. Entre les mailles de son dense réseau hydrographique, on rencontre plus d'une centaine d'espèces d'arbres dont la plupart sont très importantes pour les Xikrin puisqu'elles sont à la fois médicinales, sources d'aliments pour eux, mais aussi pour les poissons (têp) et les gibiers ( $m r y$ ) qu'ils convoitent. Les Xikrin reconnaissent une grande variété de milieux forestiers (atemãbà) dont la composition dépend de leur position dans l'espace physique, qui détermine l'hydrographie, le type de sol et de végétation, la présence de certains animaux le tout conditionnant le type d'activité qui y sera réalisé. Certaines catégories, en plus d'être reconnues et nommées, sont gérées telles que les purutum ou ibê (vieux jardins, jachères) ${ }^{8}$, pi'y-kô (castanhal, qui en portugais signifie regroupement de nombreux châtaigniers du Brésil, Bertholletia excelsa, dans une même zone) ${ }^{9}$, et kapôt $^{10}$ (les zones de cerrado qui portent le même nom en mẽbêngôkre que les pâturages) ${ }^{11}$. Il ne semble pas exister de hiérarchie de socialité des milieux en fonction de leur éloignement du village, mais en fonction de leur accessibilité et du type d'activité qu'on est supposé y faire (et donc des relations qu'on va y développer).

Loin de représenter des catégories exclusives, ces différents milieux sont plutôt perçus de manière dynamique et parfois superposée par le mouvement des hommes et leurs pratiques conditionnés par un savoir et savoir-être écologique singulier. Un après-midi, une famille se rend dans une vieille jachère à la recherche d'un tatou qui a été repéré le matin même dans la zone. Une jeune femme de cette famille emmène avec elle son bébé qui a à peine six mois et sa fille de cinq ans. Le lieu n'est pas très éloigné du village, à une demi-heure de marche à peine. Mais il faut pourtant traverser un couvert végétal diversifié composé de différents milieux, bà. Je demande à la jeune femme si ce n'est pas dangereux pour ses enfants de s'aventurer jusqu'ici, elle me répond que non, puisque le tatou est caché dans la jachère ou vieux jardin (purutym), c'est-à-dire un lieu socialisé depuis un certain temps, comme en témoigne la présence d'un grand nombre 
de bananiers. Si le tatou s'était caché dans un trou situé dans la forêt noire (bàtyk), ou forêt rouge (bàkamrek) par exemple, le bébé et la petite fille ne s'y seraient pas arrêtés, même si le lieu en question se trouvait plus proche du village car pour cela, il faut avoir atteint un certain niveau de développement physique et intellectuel dans le processus de construction de la personne.

Dans un monde totalement significatif, c'est la vie de notre corps qui donne sens aux choses qui nous entoure, tout comme ce que nous avons en tête. Selon Berque (1987, 2010), c'est la vie du corps propre qui anime le milieu, Umwelt (al.), le distinguant ainsi d'environnement, Umgebung (al.), essentiellement physique, objectivé. "Le milieu, ce n'est pas l'environnement; c'est la réalité de son environnement pour une certaine espèce ou une certaine culture, c'est-à-dire un certain environnement, spécifiquement approprié à/par cette espèce ou cette culture " (Idem). Viveiros de Castro (2007), va dans le même sens lorsqu'il avance, en se référant au concept d'affordance de Gibson (1979: 8, cité par Viveiros de Castro $2007: 4$ ), que la notion de milieu suppose un «environné ", c'est-à-dire un existant pour qui ce milieu existe puisqu'il n'y pas selon lui de milieu dans l'absolu. «Tout milieu est le milieu d'un organisme (ou d'une espèce) donné(e), pour qui, ou de qui le milieu est milieu " (Viveiros de Castro 2007, ma traduction ${ }^{12}$ ). Suivant le courant phénoménologique de l'habiter, le corps est considéré comme la condition permanente de l'expérience, puisqu'il permet une ouverture perceptive au monde et un engagement en son sein. Ceci suggère qu'il n'y a pas de vérité unique à propos de la vision du monde, mais une multiplicité de réalités à travers la perception et l'expérience du monde par le corps propre. Ainsi le milieu n'est ni donné, ni universel et sa réalité singulière ne cesse de se construire dans le rapport dynamique et réciproque d'une espèce ou d'un groupe avec son milieu de vie. L'idée selon laquelle il n'y a pas de milieu sans sujet pour qui il est milieu est, pour Viveiros de Castro (2007), très proche de la notion de perspectivisme amérindien (Stolze Lima 2005, Århem 1996, Viveiros de Castro 1996, 1998), entendu comme une multiplication de points de vue (soit, une multiplication de mondes) qui suppose des modes de représentation similaires sur des mondes différents.

Si à travers ces données, on suppose que la forêt bà est le lieu d'habitation des animaux en opposition avec krĩ le village comme lieu d'habitation des Xikrin, on pourrait être tenté d'invoquer ici les théories perspectivistes qui, en imputant à la forêt le statut d'habitat des animaux, lui assigne par la même occasion le point de vue de ces derniers au détriment des humains. Ainsi, selon cette approche, et comme le suppose Nunes (2013) à propos des Karajá du Brésil Central, lorsqu'un humain se rend en forêt, il se rend dans le territoire du jaguar et pour ne pas perdre la face en cas d'éventuelle rencontre avec le maitre de ces lieux (le jaguar), l'humain devrait adopter le point de vue du jaguar, c'est-à-dire montrer qu'il n'a pas peur. Cela impliquerait, selon Nunes, une «transformation des affects, des capacités et des dispositions des corps Karajá [c'est-à-dire une différence vestimentaire, de régime de communication, de mouvements, etc.] lorsqu'ils marchent dans la forêt ». Ainsi, Nunes argumente que « les lieux et les personnes se constituent réciproquement de sorte que les lieux stabilisent une relation dans la perspective des êtres pour lesquels ce lieu est un village ou un territoire » (Idem : 135).

Je partage avec cet auteur l'idée selon laquelle les lieux et les personnes se constituent réciproquement, comme nous le verrons plus bas, en revanche, mes données ne rejoignent pas certaines observations de Nunes, à savoir que « les lieux stabilisent une 
relation dans la perspective des êtres pour lesquels ce lieu est un village ou un territoire " (Idem : 135). D'après mes propres observations et interprétations des discours des Xikrin relatifs aux espaces forestiers et à leurs habitants, il me semble que le domaine forestier dans son ensemble est le substrat de toute approche perspectiviste et pas seulement de celle d'un de ses habitants emblématiques tel que le jaguar ou le pécari. La forêt bà est en fait ce que l'on veut qu'elle soit de son propre point de vue, et pour les Xikrin, elle est déjà plurielle, simultanément et potentiellement et surtout, productrice d'effet. En revanche, pour parler d'une «forêt qui ne sert à rien », ou " qui ne produit rien ", qui est "sans effet " parce qu'il n'y a rien à en tirer, bàmỳnha 'õ kêt, donc pas utile, pas efficace, les Xikrin emploient le terme bàkaigô. Quelque chose qui est kaigô est quelque chose qui n'atteint pas les attentes, ce qui implique que le mot qui le précède est incomplet ou inactif. Par exemple, quand quelque chose est donné (ãrã) sans contrepartie (ou sans effet), cela se dit ãrãkaigô (gratuit) ce qui est considéré comme un échange sans effet, qui ne produit rien de nouveau en retour. C'est donc finalement le terme Bàkaigô, qui peut nous en apprendre le plus, par opposition, sur ce qu'est la forêt pour les Xikrin. Et nous verrons que pour être efficace, c'est-à-dire non kaigô, la forêt bà doit produire de la valeur par l'appropriation et l'apprentissage.

\section{Les chemins comme marque d'appropriation}

Se déplacer (en forêt) se dit me y (ou me yryten) en mẽbêngôkre, où me est " gens ", $y$ est " graine » ou "semence», et ten est " marcher ». Yry est utilisé pour dire "aller à la rencontre de ", mais yry est aussi synonyme de "devenir", comme dans ba i mejyry «j'irai bientôt mieux». Mey est une manière très poétique que les Xikrin ont d'exprimer le mouvement dans l'espace, comme action organique, donnant forme à cette action par la création de chemins et de milieux, être en devenir puisque « donner forme, c'est la vie » (Klee 1973 cité par Ingold 2012 : 26), « ainsi, comme la plante croît à partir de sa graine, la ligne croit à partir du point qui a été placé dans le mouvement " (Ingold $2012: 26$ ). « Canaux conducteurs de toute activité » (Weiner 1991 cité par Ingold 2007), les chemins travaillés à chaque passage sont donc le reflet de l'appropriation de l'espace et des ressources du territoire (Figure 4). 
Figure 4 : Une femme allant en forêt pour y cueillir des fruits. Le chemin est très perceptible

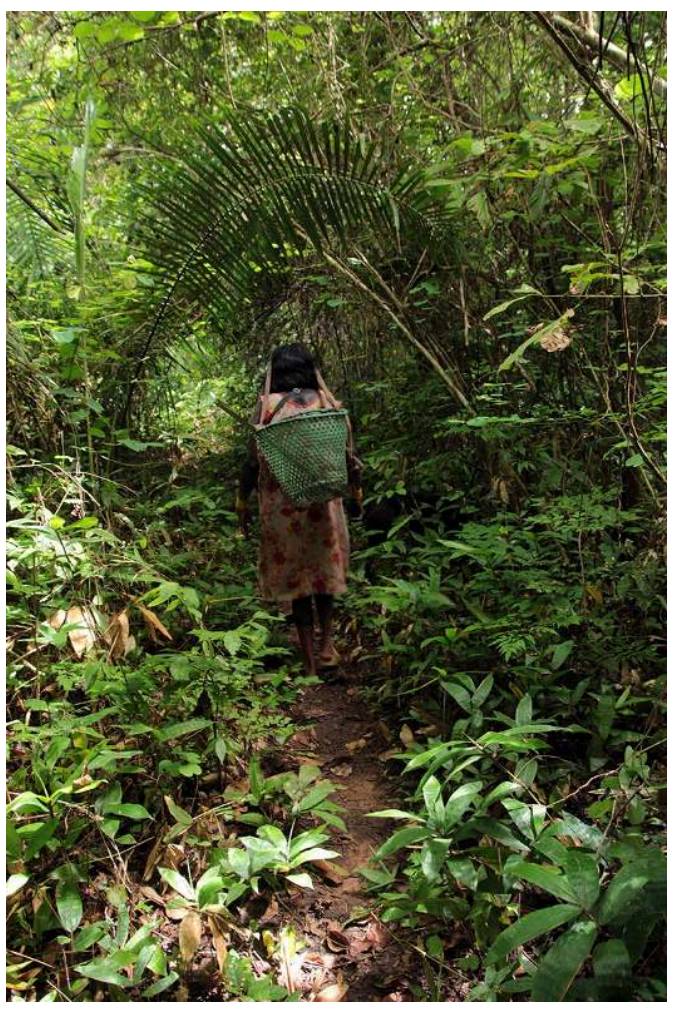

(c) S. Tselouiko, Bacajá 2015

21 Quand quelqu'un «va en forêt » casser des noix du Brésil, par exemple, s'il exploite un pied qui n'appartient à personne, et qu'en cassant l'une des coques, il découvre une noix particulièrement grande ( $y$ tire), il décrètera alors que ce pied lui appartient. De retour au village, il montrera à tout le monde la noix découverte en situant le lieu où il l'a trouvée et il déclarera «inhõ õ bàri» (mon pied). La règle est la même lorsqu'il s'agit de coco de babaçu. Tandis que pour le génipa, c'est la découverte même du pied qui établit l'appropriation, à condition bien sûr, qu'il n'appartienne pas déjà à quelqu'un.

L'appropriation d'un pied peut se matérialiser par l'inscription d'un symbole (souvent une croix, ou même dans certains cas à présent, du nom du propriétaire), sur l'écorce de l'arbre à l'aide d'une machette, mais c'est surtout le chemin qui mène au pied et le fait de nettoyer la végétation aux alentours qui marque visiblement cette appropriation. Il en va de même pour l'appropriation d'un jardin, dont le lieu est établi par l'ouverture d'un chemin sur tout le pourtour de la zone sélectionnée par le couple avant d'être nettoyé.

Les noix du Brésil sont, au même titre que les jardins, possédées par un couple plutôt que par une personne, tandis que les palmiers de babaçu sont possédés par les femmes et les génipas par les hommes, bien que ce soit les femmes qui l'utilisent exclusivement. Car dans la mesure où cette essence est très dispersée dans le territoire, ce sont plus souvent les hommes qui les rencontrent durant leurs expéditions de chasse qui sont l'occasion d'ouvrir de nouveaux chemins. L'appropriation par une personne d'un grand nombre de pieds est hautement valorisée socialement. Dans le cas de la Noix du Brésil, elle permet un meilleur retour financier par la vente des noix, puisque la rente est le principal objectif de son exploitation, même si les Xikrin l'utilisent aussi pour la cuisine, mais en bien moindre mesure. Le palmier babaçu et le génipa ont une valeur 
attribuée à l'esthétique (la beauté et la force des corps) : le babaçu permet de fabriquer de l'huile dont les femmes s'enduisent les cheveux et le corps en le mélangeant à du roucou, et le génipa sert à la peinture corporelle. Une femme qui possèdera un plus grand nombre de pieds de génipa sera plus en mesure de peindre ses enfants et ellemême. Cette capacité de production esthétique a sans aucun doute un effet sur le prestige qu'elle aura auprès des autres femmes, sachant l'importance attribuée à la peinture corporelle dans la fabrication et le langage des corps chez les Mẽbêngôkre.

Figure 5 : Un couple de mẽbêngêt et leurs filles mẽkratymre et mẽkranyre se rendent en forêt casser des noix de babassu de leur possession

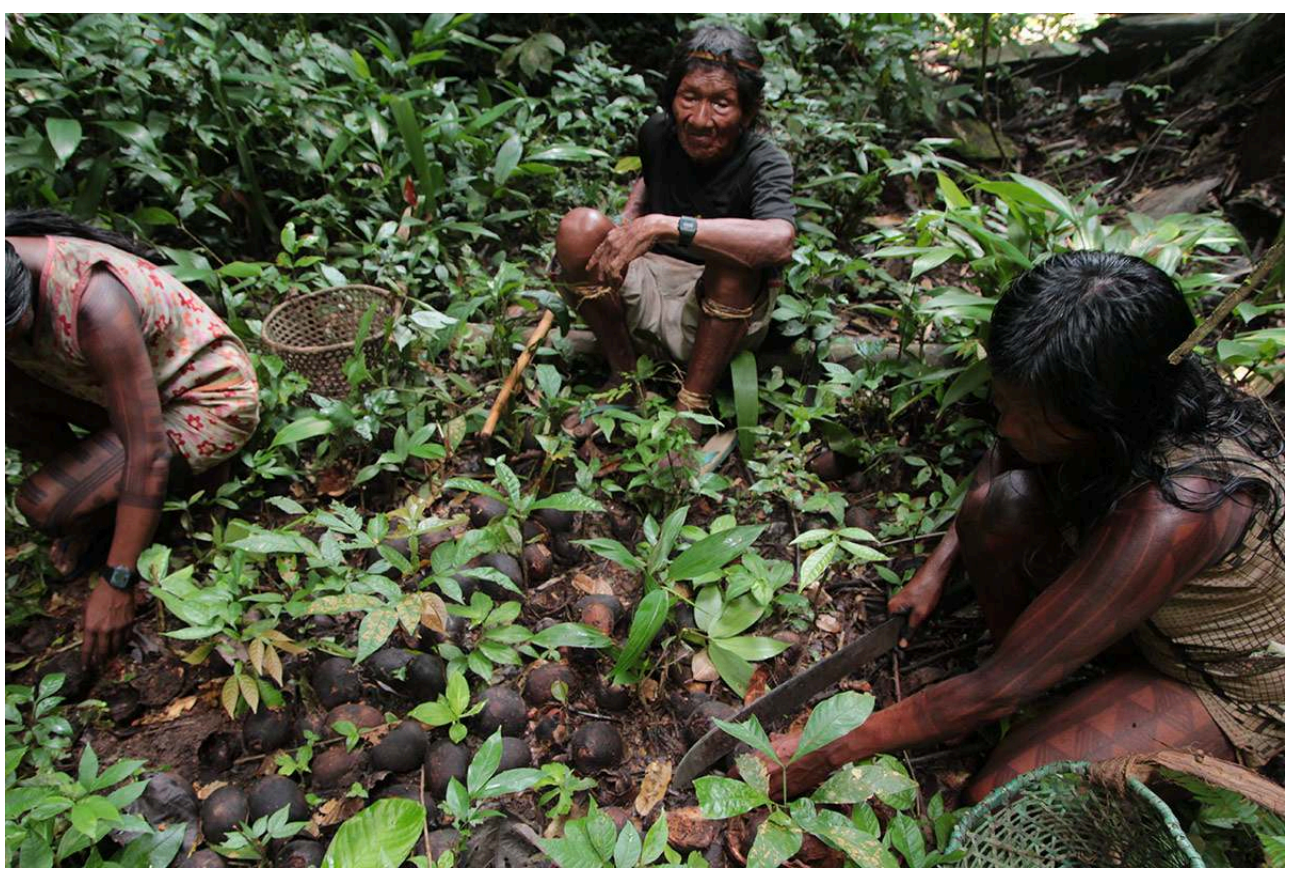

(c) S. Tselouiko 2015

On comprend ainsi mieux pourquoi les pieds de ces trois essences sont possédés de manière exclusive. Nul ne peut exploiter les pieds de quelqu'un sans son autorisation. Quand le propriétaire du pied devient trop vieux, ses enfants et petits-enfants enfants vont l'exploiter à sa place. En retour, ils pourront conserver pour eux une part de la collecte. Ce sont les plus âgés qui possèdent généralement ces essences, les jeunes gens accompagnent leurs aînés pour les exploiter, jusqu'à ce qu'ils aient atteint l'âge (ou le statut) pour devenir à leur tour propriétaire, ce qui implique en général d'avoir plusieurs enfants ou petits-enfants (Figure 6). 
Figure 6 : Collecte de noix du Brésil en famille

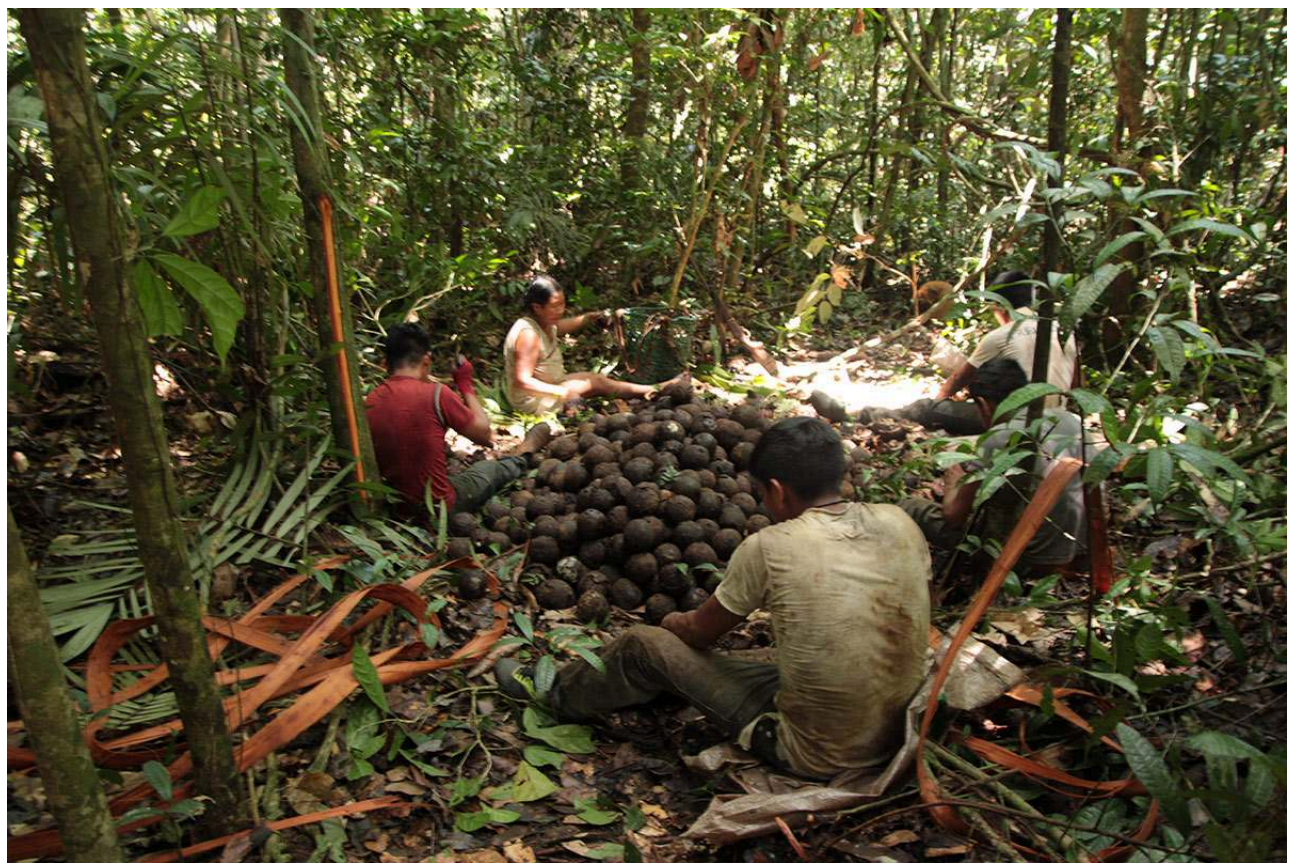

Un homme et son épouse collectent des noix du Brésil (pi-y) dans la forêt proche du village Bacajá, dans la même zone que son frère et sa belle-sœur, accompagnés de leurs fils adultes célibataires (c) S. Tselouiko 2015

Quand le propriétaire meurt, le pied possédé reste à l'époux ou à l'épouse, mais pas aux enfants, car il ne peut y avoir de propriété collective d'un pied. Quand celle-ci décède à son tour, le pied redevient d'usage commun jusqu'à ce qu'il tombe un jour, et cette chute de l'arbre est directement associée par les Xikrin à la mort du dernier propriétaire. En effet, comme me l'explique Bepdjàti, l'un des hommes les plus âgés du village Bacajá, quand le propriétaire meurt, le noyer pi-y lui manque et meurt aussi. Dans un endroit proche du village de Bacaja, il y avait beaucoup de pi-y. Tous les anciens propriétaires de ces pi-y sont morts de maladie au contact avec les Blancs, et tous les pi-y sont tombés (Bepdjàti 2014).

L'appropriation ne concerne pas seulement les essences d'arbre, mais aussi des zones plus ou moins étendues ${ }^{13}$. De la même manière que des essences spécifiques, les zones s'approprient à partir d'une découverte. Comme me l'expliqua Bepàmati, les gens se déplacent dans la forêt, ils arrivent à un endroit où le bà est bon, où il $\mathrm{y}$ a beaucoup d'animaux. L'endroit où il y a le mrôti, en amont, où il y a beaucoup de bàkamrêk; c'est le bà de Maradona, Maradona s'en occupe. On ira au bà de Maradona. Maradona fait le tour de son bà, et pour indiquer que c'est à lui il gratte son nom sur les arbres (Bepàmati 2015).

27 Sur le plan linguistique, la relation d'appropriation peut s'apparenter à celle de domestication : ron ne bao ó bàri, pi-y ne bao õ bàri = je fais le ron/pi-y mon arbre, je le fais mien, idem quand on domestique un animal $=$ baoinhôkrit $=$ je le fais mon animal domestique. Lorsque quelqu'un parle d'un arbre ou d'une parcelle qui lui appartient il dit ba ã wajêtanẽ où wajêt signifie «être suspendu ». Cette proposition que l'on pourrait traduire comme « je suis suspendu au-dessus de [ce qui m'appartient]» pourrait être prise comme une métaphore pour parler de surveillance ou de vigilance, puisqu'être 
suspendu revient à être dans une position plus haute, over see, avoir une vue d'ensemble, comme pourrait l'avoir un maître du gibier omniscient.

Cette appropriation ne doit pas être confondue avec la conquête d'un espace "sauvage » extra-villageois, mais plutôt comme une incorporation de cet espace dans le monde domestique des Xikrin, repoussant non pas les limites du social, mais les limites avec le monde inconnu (inexploré), au-delà de ces marques d'appropriation que sont les chemins, à mesure que l'on s'engage dans la forêt. Or, on ne s'engage pas dans la forêt de la même façon selon son état et en particulier selon son âge, puisqu'à mesure que la personne grandit, elle va pénétrer chaque fois plus et toujours plus loin, l'espace extra-villageois dont les signaux seront enseignés, incorporés et retournés vers cet espace par le biais de la technique. L'appropriation se fait donc par l'apprentissage continu en immersion de l'« organisme-personne » (Ingold 2000) dans ce monde dirigé à travers les chemins qui agissent ici comme écriture dans le paysage, parlant des Xikrin dans l'espace par l'espace, où non seulement l'on passe, mais surtout où l'on grandit, où l'on apprend, où l'on se produit comme Mẽbêngôkre concrètement avec le territoire. C'est pourquoi je propose de croiser cette conception de l'apprentissage avec celle de la territorialité pour affirmer le caractère dynamique et rétroactif de cette dernière à la fois sur les individus de manière différentielle et sur le groupe dans son ensemble, au lieu de la concevoir comme un simple produit de la socialité humaine. Pour explorer cette piste, abordons à présent la notion de l'apprentissage Xikrin en contexte d'immersion forestière.

\section{Amakre an tẽn, « apprendre en marchant »}

Selon Cohn (2000), l'apprentissage Xikrin va de pair avec le renforcement progressif du lien entre le karon (sorte de double, communément traduit par âme, esprit) et le corps (composé de la peau, de la chair et des os). De manière générale, apprendre progressivement se dit en langue mẽbêngôkre amakre an tẽn, "apprendre en marchant", soit, apprendre dans le mouvement, même si cela ne suppose pas nécessairement un déplacement physique, comme apprendre progressivement la peinture corporelle, ou bien la confection d'artefacts. Mais dans le cas de la forêt, cela suppose que l'apprentissage se réalise dans le déplacement à la rencontre des divers éléments du milieu, recevant les "signaux de vie », puisque l'information, comme dit Bateson (1980), existe seulement à travers le mouvement de qui perçoit son milieu. Ainsi, l'apprentissage est vécu par les Xikrin non seulement comme la participation de l'être au monde (Cohn 2000), mais aussi comme la participation du monde dans l'être.

$\mathrm{Au}$ même titre que l'apprentissage, l'engagement dans le monde forestier est différentiel, progressif et sans réelle contrainte sociale, bien qu'il soit toujours incité. C'est finalement l'expression du désir d'acquérir de nouvelles connaissances et de nouvelles aptitudes qui motive les déplacements en forêt. Un jeune homme célibataire (mênoronyre) va progressivement accompagner à la chasse, ses pères et ses frères (réels et classificatoires), ses oncles ou amis, membres de la même classe d'âge. Au début, ils n'emportent pas d'arme, mais seulement leur corps et leurs sens réunis pour apprendre à reconnaître les traces laissées par les différents gibiers et les fruits qu'ils mangent pour savoir comment les traquer. Une adolescente va principalement s'engager dans l'univers des jardins avec ses mère(s), grands-mères, sœurs et belles-sœurs, pour participer à la plantation, entretien des jardins et collecte, puis ensuite, à la 
préparation des produits récoltés. Mais à certaines époques de l'année bien précises, elle pourra aussi bien accompagner les femmes plus âgées dans leurs expéditions de collecte d'açai (kamerekà), de cocos babaçu (ron). À mesure que les jeunes gens vont parcourir les chemins, ils vont apprendre à identifier les différents parcours et les possibilités de rencontres que ceux-ci leur offrent, selon les essences présentes. Ils vont aussi apprendre quels sont les palmiers babaçu et les noyers du Brésil qu'ils ont le droit de collecter, puisque chaque pied de ces essences possède un propriétaire humain unique (et éventuellement, par extension, sa famille) en droit d'exploiter le pied en question.

31 Au retour d'une expédition de collecte en forêt, un couple de personnes âgées (mêbêngêt ) et leur fille célibataire d'une quarantaine d'années (mêkratymre) avaient repéré un tatou (Dasypus sp.) à quelques minutes à pied du village. La nuit allait tomber, ils savaient que leurs chances de capturer l'animal étaient assez faibles à ce moment-là, sachant que la capture peut durer un bon moment. Ils décidèrent alors de bloquer toutes les issues du terrier et de revenir le lendemain dans la journée (Figure 7). Le jour suivant, ils se rendirent au lieu du terrier, accompagnés d'un jeune couple sans enfant (dont l'époux est le petit-fils du couple de mêbêngêt) et un adolescent (fils de la mêkratymre). Si la présence de ces derniers pouvait bien sûr s'avérer utile, elle ne constituait pas pour autant une réelle nécessité pour la capture de l'animal, et encore moins pour le rapporter au village étant donné qu'au contraire d'un pécari ou d'un tapir, le tatou est de petite taille. Ils étaient simplement venus participer à la capture et apprendre la technique. La capture de l'animal requiert un certain savoir-faire et surtout une bonne connaissance éthologique et botanique. Les tatous creusent des galeries souterraines pourvues de nombreuses sorties espacées de plusieurs mètres. Il faut donc pouvoir toutes les repérer pour les boucher avant de localiser l'animal dans la galerie et de creuser un trou au-dessus de lui. Une fois l'animal cerné, pour l'extirper de son trou, les Xikrin emploient des plantes qu'ils font brûler pour étourdir l'animal et l'assommer à coups de bâton. Malheureusement, quand le groupe arriva au niveau du terrier où le tatou avait été retenu prisonnier la veille, ils constatèrent qu'il s'en était échappé. Mais cela n'allait pas empêcher les mêbêngêt et la mêkratymre de continuer leur excursion. Dépassant leur déception, ils décidèrent de poursuivre plus loin sur le chemin qui leur offrirait d'autres occasions de récolte et peut-être même de chasse, tandis que le jeune couple et l'adolescent rebroussèrent chemin vers le village. Le groupe se divisa, chacun dans sa direction, et ceci ne parut poser aucun problème. Chemin faisant ou pas, les trajectoires et les rencontres sont toujours conjoncturelles. 
Figure 7 : Couple de Mẽbêngêt à la recherche d'un tatou emprisonné la veille dans son trou

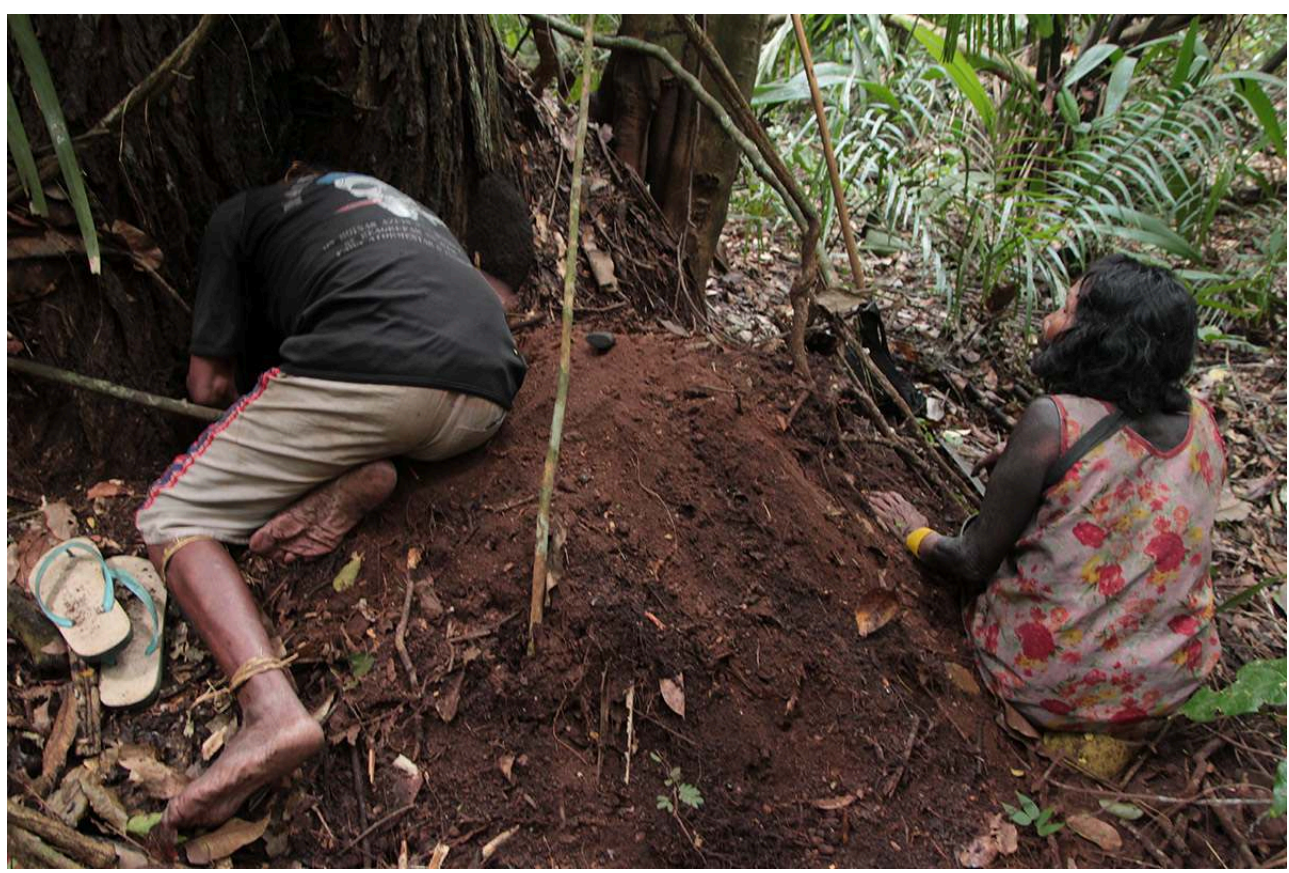

(C) S. Tselouiko 2015

Si l'engagement dans le monde forestier est différencié selon l'âge, il peut aussi l'être selon le sexe. À noter que cette différentiation n'est pas systématique, puisqu'il existe de nombreuses occasions où des groupes mixtes partent collecter des essences forestières (notamment les noix du Brésil qui je le rappelle sont appropriées par les couples). Et lorsqu'il y a différentiation, elle ne se joue pas dans le rapport exclusif d'un sexe à un type d'espace, mais plutôt dans la façon dont ce même espace peut être parcouru par les deux sexes, puisque les chemins empruntés pour chasser sont les mêmes que ceux qui mènent aux jardins ou aux différents lieux de collecte. Selon le type de ressource recherché, les femmes passeront plus ou moins de temps le long des chemins. Par exemple, si elles sont à la recherche d'açai, elles passeront la plus grande partie de leur temps dans les açaizais (lieux où se concentrent les palmiers açais), hors des chemins qu'elles n'emprunteront que pour rejoindre d'autres açaizais. Les hommes, quant à eux, marchent le plus souvent sur les chemins recherchant des traces de passage de gibier, tels que des fruits mordus, ou encore des chemins tracés par leurs passages répétés, et où ils doivent bien se garder de marcher pour ne pas laisser leur odeur qui ferait fuir les animaux. C'est seulement au moment de la poursuite d'un gibier que les hommes sortent des chemins, ou encore lorsqu'ils chassent à l'affût du haut d'un arbre fruitier dont ils savent que certains gibiers s'alimentent.

Pour résumer, l'espace de vie des Xikrin est constitué par une succession d'actions individuelles et collectives de génération en génération, qui module corrélativement la constitution d'un système de valeurs déterminant de manière concrète et singulière la compréhension du monde. Les excursions des Xikrin en forêt ne tendent pas vers une destination prédéterminée, elles n'ont pas forcément de finalité absolue, mais elles sont faites de mouvements matérialisés par les chemins qui reflètent un mode singulier d'appropriation de l'espace et des ressources. Toute excursion en forêt est l'occasion pour chaque personne, tout au long de sa vie, de faire de nouvelles rencontres avec des existants non-humains et des découvertes qui participeront à révéler toujours plus la 
richesse du milieu, tout en forgeant la connaissance de celui ou celle qui s'y déplace. En d'autres termes, les mêmes chemins connus de tous et parcourus des centaines de fois dévoilent leur caractère singulier à chaque nouveau passage. Il est alors possible d'assimiler ce processus phénoménologique de l'apprentissage territorial, à celui de l'apprentissage oral dont le caractère répétitif (Detienne 1985) et le contexte d'énonciation et de performance (Sanchés-Parga 1988 in Cohn 2006) permettent la variabilité des contenus.

C'est à mon sens ce qui définit la territorialité, entendue comme l'ensemble des rapports existentiels et sociaux que les individus en groupe et individuellement entretiennent avec l'espace qu'ils produisent et reproduisent perpétuellement de manière réciproque avec la production de leur société, contrairement à l'approche objectivée de " territoire " qui évoque plus une notion juridique moderne d'appropriation de l'espace réifié et défini par des frontières. Surrallés (2004) invoque une plasticité du territoire qui se comprend si l'on considère que la conception de l'espace se fonde sur l'état interne du sujet perceptif et ses motivations à se déplacer dans cet espace. Or, l'histoire des Xikrin est marquée par des évènements qui ont considérablement transformé leur manière d'habiter ce milieu, à commencer par le contact avec les agents du SPI ${ }^{14}$ à la fin des années 1950 et la sédentarisation au bord de la rivière Bacajá ${ }^{15}$.

Les Xikrin disent qu'avant le contact avec la société nationale (et en conséquence leur sédentarisation), les anciens marchaient continuellement dans la forêt. Cette mobilité se justifiait, entre autres, par les guerres intertribales qui sévissaient dans la région, mais pas seulement. La mobilité se pratiquait aussi par goût ${ }^{16}$, lorsqu'elle ne se réalisait pas pour l'exploitation des ressources plus éloignées. Ils marchaient beaucoup (à ten à ten à ten...) et au long de ces pérégrinations, ils réalisaient des collectes et des chasses qui se consommaient durant des fêtes pour diverses occasions en des lieux donnés, avant de poursuivre leur parcours vers un nouveau « lieu bon pour s'établir " ( $\mathrm{mmio} k \mathrm{ka}$ kimo) un temps. À partir du moment où les Xikrin ont été sédentarisés, les sorties en forêts se sont faites plus rares et plus courtes. S'ils continuent depuis à réaliser des treck de quelques semaines, ils reviennent systématiquement au même village devenu permanent où ils ont accès à une assistance médicale occidentale dont ils sont devenus dépendants. Ce nouveau mode de vie a eu un effet sur le mode d'apprentissage territorial et sur la logique d'appartenance.

De même, avant la sédentarisation, il n'y avait pas d'appropriation individuelle ou familiale d'essences d'arbre ou d'espace. Les cultivars, quant à eux, étaient bien appropriés, et ont d'ailleurs toujours constitué un bien extrêmement valorisé auprès des femmes, leurs propriétaires. Mais les jardins avaient un statut collectif, assurant au groupe tout entier autant de lieux hospitaliers où s'arrêter et manger au cours des pérégrinations (Posey 1985, 2002).

À présent, si les personnes ne se répartissent plus dans l'espace et le temps comme avant le contact, ce sont les lieux qui se répartissent entre les personnes pour permettre une "gestion territoriale " adaptée à la sédentarisation, c'est-à-dire un mode d'accès et d'usage des espaces et des entités végétales et animales du milieu, encadré par des règles sociales. Mais cette appropriation ne se réalise pas de manière uniforme sur l'ensemble du territoire, elle est toujours relative au point d'ancrage des individus : le village. Aussi, si l'on représente les parcours forestiers des Xikrin dans un rayon de cinq à dix kilomètres autour du village, on observe une structure réticulaire 
de l'occupation spatiale qui contraste avec la représentation en zonage concentrique souvent invoquée pour traiter de la socio-spatialité en Amazonie, ce que Albert et Le Tourneau (2007) ont d'ailleurs parfaitement bien démontré chez les Yanomami. Pour aller dans le même sens, la figure 8 représente une partie des chemins parcourus quotidiennement par les Xikrin du village Bacajá à l'époque où je réalisais mon terrain de novembre 2014 à septembre $2015^{17}$. Ces parcours ne concernent que la cueillette, puisque les expéditions de chasse m'étaient plus difficiles à accompagner ${ }^{18}$. À la lecture de cette carte, on peut aisément remarquer deux choses. Premièrement une structure réticulaire et radiale qui indique une exploration raisonnée et opportuniste de l'espace et deuxièmement, un contraste entre lignes et cercles, à savoir entre chemins et zones d'extraction, qu'il sera important de garder à l'esprit pendant l'analyse des modes de transcription cartographique de l'usage de l'espace forestier.

Figure 8 : Chemins forestiers proches du village Bacajá

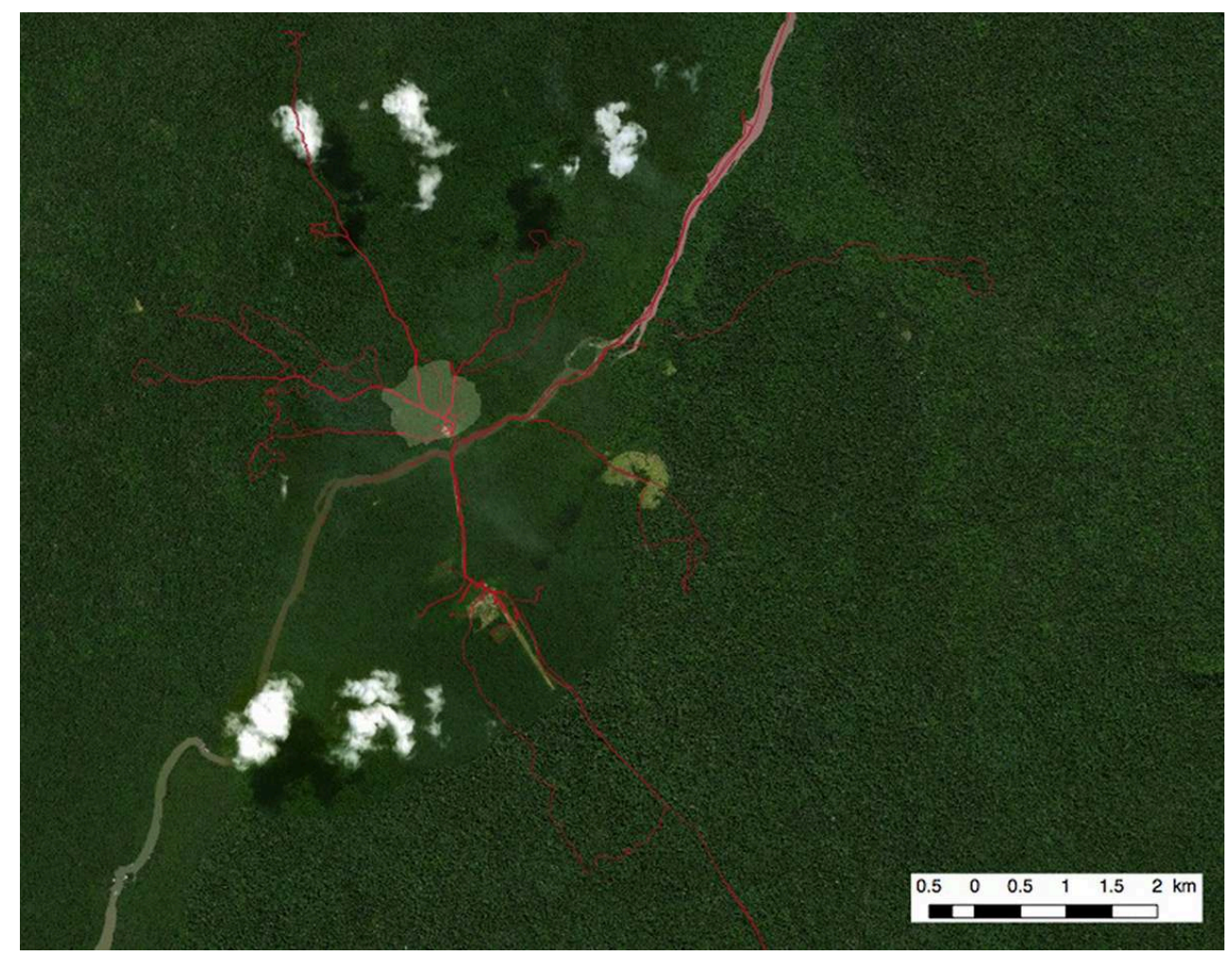

Image réalisée à partir du tracé GPS enregistré à chaque excursion que l'auteure a réalisé durant le terrain

Les bà appropriés sont des lieux de chasse aisés, près du village, où l'on ne met pas plus de deux heures pour y aller, les bà plus éloignés sont plus difficilement appropriables individuellement. Tandis qu'à plus grande échelle, ces espaces forestiers éloignés des villages sont perçus comme la propriété d'un collectif, comme lorsque les Xikrin parlent de leur territoire en ces termes : gwajbanhõbà (gwajbanhõ = notre ; bà = traduit souvent comme forêt, mais ici porte le sens de milieu), ou encore lorsqu'ils parlent du territoire des Assurini : kukenbrãjakàrenhõbà (kukenbrãjakàre= Assurini).

Passant d'un mode de vie semi-nomade à sédentaire, c'est l'adoption de cette logique d'appropriation qui a permis progressivement aux Xikrin d'articuler leurs savoirs territoriaux avec la conception juridique moderne de territoire pour finalement 
obtenir la démarcation de leur terre en 1996. Aujourd'hui, avec les projets de développement qui surgissent dans le paysage des Xikrin, leur espace de vie est amené à être encore plus objectivé à travers la mise en place d'un projet de gestion environnementale et territorial ${ }^{19}$ indigène qui comprend notamment la réalisation d'ethnocartes, dans le cadre de la Politique Nationale de Gestion Environnementale et Territoriale Indigène - PNGATI.

Korzybski (2007) nous dit qu'« une carte n'est pas le territoire », mais un langage qui reflète dans sa structure une vision du monde tel qu'il est conçu. C'est une représentation subjective d'une vision du territoire, à l'image de ses auteurs. Mais dans un contexte où différents régimes de savoirs et perceptions spatiales se rencontrent pour élaborer des cartes, en employant, pour y parvenir, une méthodologie totalement étrangère à l'auteur principal (ici, les Xikrin), il s'agit de se demander comment ce type de projet permet-il de mettre en récit des expériences individuelles du territoire sous forme de cartes uniformisées, standardisées? Ces dernières permettent-elles réellement de refléter un savoir territorial local, c'est-à-dire une «ethnocartographie», ou bien sont-elles à l'origine non seulement d'un nouveau savoir territorial, mais aussi d'une nouvelle conception territoriale? Pour illustrer cette problématique, place à présent à l'étude ethnographique d'un atelier d'ethnocartographie réalisé chez les Xikrin de 2013 à 2015.

\section{L'ethnocartographie, innovation ou révélation d'un savoir territorial ?}

La première phase du projet menée entre juillet et novembre 2013 a consisté à former de jeunes hommes Xikrin à la gestion environnementale, en commençant par l'utilisation de GPS comme préalable à l'élaboration d'ethnocartes. Après un atelier théorique qui avait pour finalité de leur expliquer le mode de fonctionnement de cette technologie en lien avec les satellites, une mise en pratique s'est concrétisée au niveau des limites orientales de la Terre Indigène, à savoir celles qui la séparent du front pionnier agricole. Les apprentis cartographes ont suivi avec leur GPS une partie de la ligne de démarcation orientale en profitant pour nettoyer la végétation afin de rendre visible la démarcation. À partir de cette première formation, les jeunes hommes ont été sollicités pour collecter de manière "autonome ", telle qu'envisagée par le projet, les points de «ressources importantes »dont la liste et les catégories avaient cependant déjà été fixées (points de pêche, point de chasse) à indiquer par la suite sur la carte.

En octobre 2014, ont eu lieu plusieurs ateliers dans les villages pour produire in fine, des cartes de la TITB dans son ensemble, à grande échelle, chacune transmettant des informations distinctes : l'histoire d'occupation du territoire, les ressources (collectes, pêche, chasse) qui le composent et les menaces qui y pèsent (orpaillage, pêche, extraction de bois, barrage, routes, etc.). Cette division en classes d'informations a été suggérée par les consultants, "pour ne pas désordonner une seule carte avec trop d'informations » qui la rendrait illisible. Afin de mettre en avant les particularités du territoire divisé en aires d'usage de chaque village, une carte à plus petite échelle a également été réalisée, sur laquelle les cartographes placèrent des informations plus détaillées sur les ressources disponibles dans la zone proche des villages. Sur cette carte, tous les types de ressource ont été mélangés. 

les Xikrin, représentant chacun une classe d'information distincte. Une liane représentait un point de pêche à la nivrée, un pied de frutão (Pouteriapariri) représentait un point de chasse, un pécari pour une aire de reproduction du gibier, etc. Sur les cartes ont également été marqués et dénommés les différents milieux atemãbà. En plus de les localiser, les consultants demandèrent aux cartographes de les définir les uns par rapport aux autres afin de révéler une éventuelle structure écologico-spatiale composée par l'ensemble de ces milieux. Et pour chaque milieu, il leur fût également demandé de décrire le type de sol et de végétation rencontré, d'énoncer son importance en termes d'usage, et en particulier la présence d'animaux et de plantes collectées, et enfin, de dire qui y va (homme, femme, tout le monde, etc.). Ainsi, à titre d'exemples, la forêt inondée (bua noro), est celle qui se situe au bord des ruisseaux et de la rivière et qui est recouverte d'eau la moitié de l'année, à la saison des pluies. La végétation y est basse, composée de nombreux fruitiers dont s'alimentent les gibiers et les poissons et constituent en ce sens une excellente zone de chasse et de pêche. La forêt basse (bàprire), quant à elle peut se former à différents endroits. C'est la faible profondeur de son sol qui engendre sa configuration. Elle est généralement peuplée de lianes fruitières dont les animaux viennent se nourrir et en particulier les tortues terrestres jabuti. Les personnes y vont souvent en groupe pour aller les ramasser. La forêt noire (bàtyk) basée sur la terre ferme est quant à elle une forêt haute. Son sol est profond ce qui en fait un excellent « dortoir » pour les tatous qui creusent des galeries souterraines. De nombreux arbres fruitiers attirent également une grande diversité de gibiers, complétant ainsi sa qualité de réserve de chasse. Sa terre est noire et de bonne qualité. Quand il se situe près des villages, c'est dans ce milieu que les Xikrin préfèrent y ouvrir leurs nouveaux jardins. Au contraire, la forêt rouge (bàkamrek), est parsemée de pierres et sa terre aréneuse est relativement pauvre. Elle doit son nom à la tonalité de couleur du couvert végétal jaune-orangé. Elle peut être dense (êpyti) comme très ouverte (bàkej), mais quoi qu'il en soit, les arbres fruitiers s'y font très rares. Elle n'est donc privilégiée ni pour la chasse, ni pour l'horticulture. En revanche, certaines essences forestières y sont recherchées comme le bàtpram (espèce ?), dont l'écorce sert à faire du charbon pour la peinture corporelle et le timbó (espèce ?), une liane toxique employée pour la pêche à la nivrée ritualisée, très importante dans le cycle cérémoniel et productif des Xikrin.

Bien que ces connaissances pédologiques, botaniques et zoologiques soient bien celles des Xikrin, leur mise en forme ne fait pas vraiment sens avec leur expérience de l'espace vécu, puisque comme je l'ai décrit plus haut, la perception spatio-temporelle de l'espace est en fait mobile et de nature égocentrique puisque «le lieu est fixé par l'échelle de [leur] quotidien " (Mazurek 2013 : 134), et non par une échelle absolue. Elle suppose de « reconnaître que l'action pratique a toujours un positionnement dans un « ici » et un «maintenant ", depuis où se voit l'autre, depuis où se voit d'une façon particulière le monde » (Lindón 2000 cité par Mazurek 2013 : 134). En d'autres termes, le lieu est défini en fonction du mouvement, c'est-à-dire dans la relation dynamique qui s'établit entre l'individu et son milieu. Aussi, si l'on prend chaque lieu (de collecte, de chasse, de pause, de campement, etc.), comme à la fois débouché de plusieurs lignes (chemins) et comme source de plusieurs devenirs, où des groupes peuvent se rejoindre comme se séparer pour déployer des activités différentes, l'idée de Ingold (2012) selon laquelle la maille ou le mycélium traduirait mieux le concept de «milieu » me semble appropriée pour envisager la forêt non pas comme un espace circonscrit mais plutôt 
comme une «texture du monde » où chacun y inscrit sa trajectoire croisant d'autres trajectoires dans des combinaisons diverses, ce qui fait qu'un point de cueillette n'est pas simplement un point de cueillette, il est un des possibles croisements de plusieurs trajectoires mettant en relation toutes sortes d'existants.

Le fait que les Xikrin aient malgré tout répondu à l'exercice de classification par l'identification et la dénomination des différents milieux sous forme d'" ethnozonage " d'après des critères naturalistes orientés par les consultants, suggère plus leur capacité à s'approprier un nouveau langage pour créer, que la révélation d'un savoir territorial (Harley 1989). Cette création relève d'une transformation, une codification de la forêt en carte, permise dans un contexte d'énonciation particulier, celui où une multitude d'expériences individuelles et régimes de savoir se croisent dans un cadre référentiel plus large.

Parmi les différentes catégories de milieux forestiers construits par les cartographes, il y a êpyti, qui, comme nous pouvons le constater, n'emploie pas le même préfixe (bà) que les autres. $\hat{E}$ en mẽbêngôkre signifie végétation (comme celle que l'on doit nettoyer régulièrement dans les jardins, " purukamêre » - êre : retirer la végétation, désherber) et pyti veut dire lourd, dense. C'est aussi le seul milieu que les Xikrin disent ne pas pénétrer, même s'il se situe dans un périmètre proche du village car, comme son nom l'indique, il est trop dense et peuplé de lianes et de plantes épineuses, c'est un milieu qu'ils contournent seulement. Lors de la restitution des cartes produites par les jeunes cartographes Xikrin (Figure 9), l'un d'eux présenta une des zones forestières comme bàkapryt "forêt vide ", à savoir "vide d'humains " car selon ses explications, si les Anciens s'y rendaient avant, aujourd'hui les gens n'y vont plus, markêt, ils ne savent pas y aller, ils ne savent pas quoi y trouver, bien qu'elle représente quasiment la moitié du territoire. Cette zone s'est vue redéfinie par ces jeunes cartographes comme une zone de reproduction du gibier, mry te ami kamkradjirekadjy, « là où les animaux vont pour concevoir ». Cela a été traduit par area mãe en portugais ("aire mère », c'est-à-dire l'aire de reproduction). Juste après, un de ces jeunes hommes indique une autre zone : " ça c'est là où on va tout le temps, c'est là où on chasse. On ne va pas de l'autre côté sinon il n'y aurait plus de gibier ». 
Figure 9 : Présentation des cartes à la communauté

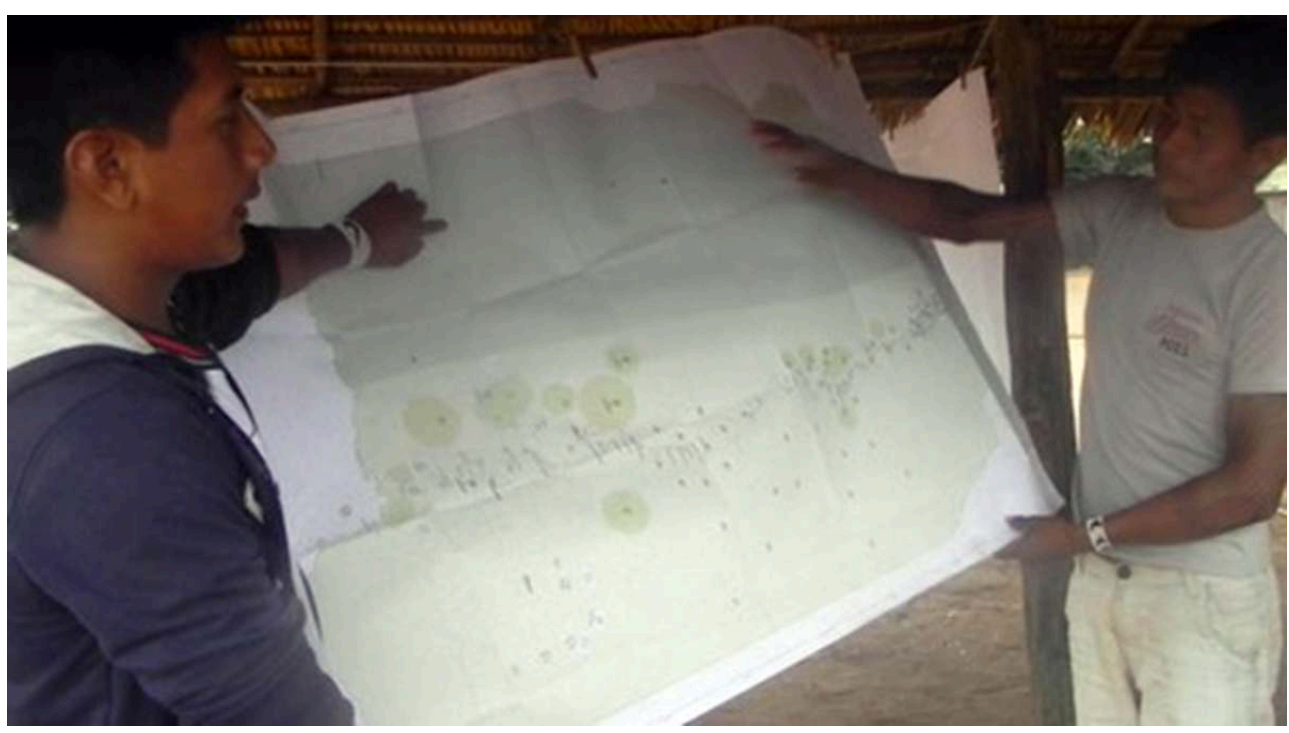

Deux jeunes hommes présentent à la communauté la carte qu'ils ont réalisée durant les ateliers d'ethnocartographie. Sur cette photo, ils pointent l'aire de reproduction du gibier

Source : Photo issue d'une vidéo réalisée par l'auteure en décembre 2014

Jeunes hommes célibataires pour la plupart, voire jeunes mariés avec des enfants en bas âge, les cartographes Xikrin furent désignés par leurs communautés pour participer à ces ateliers, car ils sont plus lettrés et parlent plus le portugais que les femmes et les aînés et sont plus familiarisés avec la manière de concevoir le monde des Kuben (les non Xikrin) et en particulier leur conception de la forêt comme une "nature sauvage " aussi bien utile d'exploiter, que vitale de préserver. En revanche, du fait qu'ils n'appartiennent qu'à deux classes d'âge (les jeunes adultes célibataires et les jeunes pères) et à un genre unique dans cet atelier, leur connaissance du territoire est partielle, a fortiori compte-tenu de leur mode de vie sédentaire qui contraste avec celui adopté par les anciens à l'époque pré-contact. Bien que les anciens et les femmes aient été incités à participer également aux discussions pendant les ateliers d'ethnocartographie, cette lacune ne peut être totalement compensée. La transformation de la forêt à une carte est contrainte par le cadre sémantique où la traduction conceptuelle, dont ces jeunes hommes sont les garants, s'ajoute au procédé de codification inhérent à la cartographie.

En passant par la réification et la création d'un nouveau savoir territorial, l'ethnocartographie a également réifié le territoire lui-même. La codification de la forêt en carte a permis la codification de la territorialité en territoire. Cette transformation apparaît clairement dans la discussion autour de l'usage politique que ces cartes devront assurer, comme permettre la " construction de scénarios concernant l'usage et la conservation du territoire ", tel qu'énoncé par le PNGATI.

\section{De la forêt au papier : usage politique des ethnocartes}

En décembre 2014, une Assemblée Générale fut organisée dans le cadre du projet GATI avec les représentants de chaque communauté de la TITB, la FUNAI et la TNC. Lors de cette Assemblée, en essayant de définir avec les Xikrin l'usage politique que ces ethnocartes devraient assurer, les consultants du projet GATI projetèrent plusieurs 
films montrant des exemples de lutte territoriale d'autres groupes indigènes du Brésil. Ils lurent aussi deux textes, dont l'un rédigé par l'organisation APIB (Articulation des Peuples Indigènes du Brésil) à propos des manifestations indigènes de 2013 contre la PEC $215^{20}$, ainsi qu'une interview de Sonia Guajajara, représentante de l'APIB. Ils présentèrent également une carte du DNPM (Département National de Production Minière), qui prévoit la prospection minière sur la totalité de la TITB. Et à partir de ces démonstrations, une longue discussion se fit sur des questions thématiques «à quoi doivent servir ces cartes ? » et «à quoi ne doivent pas servir ces cartes ? ».

Au-delà de s'approprier les nouvelles formes de figuration du territoire, les Xikrin se sont également approprié le discours d'écologie politique occidental montrant ainsi qu'ils saisirent bien le défi de créer ces cartes pour défendre leurs intérêts futurs.

51 Si nous ne prenons pas soin de la terre, ils se dépêcheront de faire sortir la loi [PEC 215] afin de détruire notre terre. Nous devons agir, surveiller, protéger pour ceux qui sont en train de naître. Aujourd'hui, nous sommes ici, mais demain nous ne le serons plus. Nous devons préparer la terre pour ceux qui sont en train de naître (Tedjere 2016, ma traduction $^{21}$.

52 Cependant, la divulgation des cartes aux Kuben avec la localisation des points de chasse, de pêche et de cueillette est apparue comme une menace à l'intégrité du territoire. Comme ils l'ont dit à plusieurs reprises, "ces cartes doivent rester quelque part au village parce que si elles sortent, elles peuvent se retrouver entre les mains de quelqu'un d'autre et ça serait mauvais pour nous $»^{22}$. Finalement, l'utilisation la plus claire que les Xikrin exprimèrent à propos des cartes était la matérialisation des invasions et la matérialisation des frontières de la TITB. Matérialisation, d'ailleurs, qu'il ne suffit pas de faire sur le papier, mais qui doit surtout être reportée in situ. En effet, les Xikrin insistèrent sur la nécessité d'élargir les chemins de délimitation du territoire, de sorte que la terre soit "vue du ciel». Cette demande explicitement formulée interpelle par la similarité du point de vue emprunté: le ciel. Cette approche surplombante est également exprimée par les Xikrin à travers les notions mẽbêngôkre wajêt qui signifie « être suspendu ", comme nous l'avons déjà vu à propos des zones de chasse appropriées qui doivent être soignées.

53 Toutefois, cette perspective d'appropriation territoriale et des ressources ne représente pas tout à fait la même chose pour les Xikrin et pour les consultants des ateliers d'ethnocartographie. Il semblerait que pour les Xikrin, il ne s'agisse pas tant d'une préoccupation pour la «protection des ressources» en tant que «bien commun de l'humanité ", mais plutôt d'une préoccupation pour la production de différence entre les personnes et les groupes à travers l'appropriation exclusive et/ou contrôlée de certaines ressources et moyens de production. Dans le quotidien des Xikrin, d'après l'exemple cité plus haut, le fait qu'un propriétaire sabote sa propre ressource lorsqu'il se rend compte qu'elle a été exploitée par quelqu'un d'autre sans son accord en est une preuve.

54 À l'échelle du groupe, la préoccupation pour la production de différence est également vraie. La dimension d'appartenance collective à un territoire, telle qu'elle apparait dans la définition juridique du territoire indigène au Brésil peut trouver une correspondance par l'expression Xnhõbà où $X$ est le collectif, nhõ marque la possession et bà prend le sens de territoire, plus que de forêt en tant que type de couvert végétal ${ }^{23}$. Durant un atelier d'ethnocartographie, le mêbengêt Bepàmati désigne le territoire des Arawete sur la carte satellite comme étant kubenkamretinhõbà (le bà des Arawete). Lorsque les 
consultants expliquèrent aux Xikrin que la carte devait servir, entre autres, de base pour une gestion territoriale (c'est-à-dire une utilisation raisonnée des ressources pour garantir la continuité du collectif), les Anciens présents à la réunion décrétèrent que c'est exactement ce qu'ils avaient toujours fait lorsqu'auparavant, ils menaient la guerre, entre autres, contre les Indiens Parakanã, les Arawete et les Kuben pour les expulser, s'approprier leurs jardins et leurs cultivars, conquérir de nouveaux territoires de chasse et de cueillette. Pour eux, la guerre, c'était bien de la gestion territoriale autant qu'une manière de produire de la différence.

Le temps de la guerre à proprement parler est révolu. Mais cette relation de différence a été aujourd'hui remplacée par un autre type de relation, un autre type de confrontation, la confrontation politique avec les Kuben, notamment à propos de la construction du barrage hydroélectrique de Belo Monte et de la mise en place des programmes de compensation - PBA. Depuis le retour du projet du barrage en 2010 et les études d'impacts socio-environnementaux qui l'ont accompagné ${ }^{24}$, les Xikrin enchaînent les réunions en ville et aux villages. Bien conscients des menaces qui pèsent sur eux et sur l'avenir de leurs enfants avec la construction de ce barrage, les Xikrin s'efforcent de se battre pour faire valoir leurs droits et des projets de compensation réellement effectifs.

\section{Conclusion}

Dans cet article j'ai souhaité montrer que pour les Xikrin, "aller en forêt " n'est pas simplement une question de déplacement d'un point à un autre. "Aller en forêt », c'est le mouvement même qui permet à chaque personne de se développer en accumulant pratiques et connaissances, en intégrant et en participant d'une maille interconnectant lieux et êtres humains et non humains. Ces excursions des Xikrin dans l'espace extravillageois sont faites de mouvements matérialisés par les chemins qui, conduisant toute activité, reflètent et registrent un mode singulier d'appropriation de l'espace et des ressources.

Dans cet exercice de traduction de diverses expériences territoriales en savoir cartographique, chaque expression individuelle du savoir a dû s'en remettre à un cadre référentiel plus large pour faire sens et être communiqué. Ce cadre référentiel a été fourni d'une part par les consultants, mais aussi par les anciens qui garantissaient la cohérence finale. C'est dans ce passage d'un savoir à l'autre que la création d'un nouveau savoir a été permise, savoir hybride qui est devenu essentiel dans la revendication et l'affirmation des droits d'autonomie et de gestion territoriale des peuples autochtones. En poursuivant leur formation et leur collecte de points GPS, et en présentant les cartes à chaque réunion publique au centre du village où elles seront commentées et modifiées au besoin, les Xikrin auront la possibilité de les faire vivre en même temps qu'ils élargiront leur expérience territoriale. C'est donc bien encore en suivant des chemins que l'on grandit... 


\section{BIBLIOGRAPHIE}

Aben Kaben Marimei, Plano de Gestão Territorial e Ambiental. Povo Xikrin da Terra Indígena Trincheira Bacajá, 2018, The Nature Conservancy. [En ligne :] https://acervo.socioambiental.org/ acervo/livros/aben-kaben-marimei-plano-de-gestao-territorial-e-ambiental-povo-xikrin-daterra

Albert B. \& Le Tourneau F.-M. 2007 - Ethnogeography and Resource Use among the Yanomami: Toward a Model of "Reticular Space". Current anthropology 48 (4) : 584-592.

Århem K. 1996 - The Cosmic Food Web : Human Nature Relatedness in the Northwest Amazon. In : Descola P. \& Pálsson G. (Ed.) Nature and Society: Anthropological Perspectives. London, Routledge : 185-204.

Bateson G. 1980 - Vers une écologie de l'esprit 2. Paris, Seuil, 352 p.

Berque A. 1987 - Écoumène. Introduction à l'étude des milieux humains. Paris, Belin.

Berque A. 2010 - La biodiversité comme impératif moral. De l'histoire naturelle à une histoire humaine. Conférence. Centro Internazionale di Studiinterculturali di semiotica e morfologia, Università di Urbino. http://ecoumene.blogspot.com/2013/02/la-biodiversite-et-lordre-moral-berque.html Bonnemaison J. 1981 - Voyage autour du territoire. L'Espace géographique, 10 (4) : 249-262.

Cohn C. 2000 - A criança indigena : a concepçao Xikrin de infância e aprendizado. Mémoire de master : Anthropologie sociale et ethnologie : USP, São Paulo.

Cohn C. 2005 - Relações de diferença no Brasil Central : os Mebengokré e seus outros. Thèse :

Anthropologie sociale et ethnologie : Faculdade de Filosofia, Letras e Ciências Humanas : USP, São Paulo.

Detienne M. 1985 - La invención de la mitilogia. Barcelone, Ediciones Peninsula.

Fisher W. 1991 - Dualism and its Discontents: social organization and village fissioning among the XikrinKayapo of Central Brazil. Thèse : Anthropologie sociale et ethnologie : Faculty of the Graduate School of Cornell University.

Fisher W. 2000 - Rainforest Exchanges: Industry and Community on an Amazonian Frontier. Washington and London, Smithsonian Institution Press.

Garcia U.F. 2010 - Karawara, a caça e o mundo dos Awá-Guajá. Thèse : Anthropologie sociale et ethnologie : USP, São Paulo.

Garcia U.F. 2012 - Ka'á Watá, "andar na floresta” : caça e território em um grupo Tupi da Amazônia. Mediações Revista de Ciências Sociais 17 (1) : 172-190.

González Pérez S. E. 2011 - Produtos florestais não-madeireiros em terras indígenas Kayapó no estado do Pará: diversidade e uso. Dissertation de máster, MPEG, Belém.

Harley J.B. 1989 - Deconstructing the map. Cartographica 26 : 1-20.

Ingold T. 2000 - The perception of the environment: essays on livelihood, dwelling and skill. London, Routledge.

Ingold T. 2007 - Lines: a brief history. London, Routledge.

Ingold T. 2012 - Trazendo as coisas de volta à vida : Emaranhados criativos num mundo de materiais. HorizontesAntropológicos 37 : 25-44. 
Korzibsky A. 2007 - Une carte n'est pas le territoire. Prolégomènes aux systèmes non-aristotéliciens et à la sémantique générale. Paris, l'Éclat, 192 p.

Malmberg T. 1980 - Human Territoriality: survey of behavioural territories in man with preliminary analysis and discussion of meaning. The Hague, Paris, New York, Mouton Publishers.

Mantovanelli T.R. 2016 - Os Xikrin da Terra Indígena Trincheira Bacajá Eos Estudos Complementares do Rio Bacajá : reflexões sobre a elaboração de umlaudo de impacto ambiental. Horizontes Antropológicos 46 : 159-188.

Mazurek H. 2013 - Cartographie : vision ou reflet ? Une réflexion autour des « références indigènes ». Information géographique 77 (4) : 109-148.

Nunes E.S. 2013 - O território das onças e a aldeia dos brancos: lugar e perspectiva entre os karajá de buridina (Brasil central). Journal de la Société des Américanistes 99 (2) : 135-164.

Posey D.A. 1985 - Indigenous management of tropical forest ecosystems: the case of the Kayapo indians of the Brazilian Amazon. Agroforestry Systems 3 : 139-158.

Posey D.A. 2002 - Kayapó ethnoecology and culture. New York, Routledge, 285 p.

Robert P. de \& Laques A.-E. 2003 - « La carte de notre terre ». Enjeux cartographiques vus par les indiens kayapó (Amazonie brésilienne). Mappemonde 69 : 1-6.

Robert P. de 2004 - « Terre coupée » Recomposition des territorialités indigènes dans une réserve d'Amazonie. Ethnologie française 34 : 79-88.

Robert P. de 2009 - Del pi'y-kô al bosque certificado, los varios caminos de lacastanha. Anuario americanista europeo 6-7 : 563-581.

Robert P. de, Lopez Garces C., Laques A.-E. \& Coelho Ferreira M. 2012 - A beleza das roças : agrobiodiversida de Mebêngôkre-Kayapó em tempos de globalização. Bol. Mus. Para. Emílio Goeldi. Cienc. Hum. 7 (2) : 339-369.

Stolze Lima T. 2005 - Um Peixe Olhou Para Mim. O povo Yudjá e a perspectiva. São Paulo, UNESP/ ISA/ NUTI.

Surrallés A. 2004 - Horizontes de intimidad. Persona, percepción y espacio en los Candoshi In : Surrallés A. \& Garcia Hierro P. (Ed.), Tierra adentro. Territorio indígena y percepción del entorno : 137-162.

Verswijver G. 1992 - The club-fighters of the Amazon. Warfare among the Kayapo Indians of Central Brazil. Gent, Rijkuniversiteit, 378 p.

Vidal L. 2007. 1977 - Morte e Vida de uma Sociedade Indígena Brasileira : os Kayapó-Xikrin do rio Cateté. São Paulo, Hucitec/Edusp, 268 p.

Viveiros de Castro E. 1996 - Os pronomes cosmológicos e o perspectivismo ameríndio. Mana 2 (2) : $115-44$.

Viveiros de Castro E. 1998 - Cosmological deixis and amerindian perspectivism. The Journal of the Royal Anthropological Institute 4 (3) : 469-488.

Viveiros de Castro E. 2007 - A natureza em pessoa: sobre outras práticas de conhecimento. Encontro «Visões do Rio Babel. Conversas sobre o futuro da bacia do Rio Negro ». Instituto Socioambiental e a Fundação Vitória Amazônica, Manaus, 22 a 25 de maio de 2007. 


\section{NOTES}

1. Cet article est rédigé dans le cadre du projet « Configurations socio-spatiales, défis politiques et débats ontologiques en Amazonie », projet financé par l'Agence nationale de la recherche ANR-17-CE41-0013

2. Il existe une certaine confusion dans la littérature entre les ethnonymes Mẽbêngôkre, Xikrin et Kayapó. D'après les recherches historiques et ethno-historiques, ces groupes seraient issus du même groupe ancestral, les Gorotire-Kumren. Ils partagent en commun de nombreux traits culturels (structure politique et économique, organisation socio-spatiale, activités de subsistance, rituels, peintures corporelles, etc.), de nombreux mythes, et parlent la même langue mẽbêngôkre (appartenant à la famille linguistique macro-Jê), avec certaines nuances linguistiques d'un groupe à l'autre.

3. Le contact s'est réalisé par des agents du SPI - Serviço de Proteçãoaos Índios (Service de protection aux Indiens), Institution précédant l'actuelle FUNAI - Fundação Nacional do Índio (Fondation nationale de l'Indien) avec l'aide de quelques Kayapó également locuteurs de la langue Mẽbêngôkre, pour faciliter le contact. Les nouvelles techniques de pêche et la navigation ont été transmises par les agents du SPI (puis FUNAI) qui ont vécu au sein de la communauté jusqu'en 2009.

4. À l'époque où je réalisais ma recherche de terrain, entre 2013 et 2015 la population comptait à peine 1000 personnes et se répartissait dans neuf villages. Depuis mon départ, le nombre de villages a presque doublé et la majorité se situe surtout au nord, à la fois plus proches des limites de la TI et plus proches des centres urbains. Ces nouveaux villages ont tous été construits au bord des routes construites en guise de compensation du barrage qui doit provoquer l'assèchement partiel de la rivière Bacajá. Parallèlement à cette reconfiguration territoriale, on observe aujourd'hui que les séjours en ville sont beaucoup plus nombreux et plus longs qu'auparavant et l'organisation socio-spatiale au sein des villages s'est aussi transformée.

5. Les Xikrin se considèrent en effet comme un peuple forestier en raison de leur histoire migratoire. Suite à leur séparation des autres groupes mẽbêngôkre, ils ont migré à l'ouest de la région de l'Araguaia caractérisée par un écosystème de savane, pour évoluer de manière seminomade sous le couvert forestier tropical du bassin de la rivière Bacajá jusqu'au contact.

6. Je tiens à exprimer ici ma très grande reconnaissance à Andrés Pablo Salanova, linguiste spécialiste de la langue mẽbêngôkre qui m'a accueilli très généreusement dans son département à Ottawa pour écouter, traduire et discuter avec moi des enregistrements sélectionnés en fonction de leur utilité pour le sujet de ma thèse. L'expertise linguistique de Salanova a permis d'adopter une approche contextualisée des discours en Mẽbêngôkre, ce qui a souvent révélé des données insoupçonnées. Ma recherche s'est considérablement enrichie grâce à ce travail.

7. Les tortues terrestres kaprã ne se tuent pas dans la forêt, elles sont capturées et rapportées au village, encore vivantes. Leur prise est plus considérée comme de la cueillette que comme de la chasse par les Xikrin. D'ailleurs ils ne disent pas à ce propos kaprãbin (chasser la tortue) comme pour la chasse du pécari (angrô bin), mais bien kaprãamu prendre la tortue avec les mains)

8. À propos des jardins mẽbêngôkre, voir Robert et al. 2012, Posey 1985 et 2002.

9. Pour une analyse des modes de gestion des pi-y kôpar les Kayapó et de leur introduction dans le marché à travers un projet d'exploitation soutenable, voir Robert 2009.

10. Kapôt est aussi le nom donné au terrain de foot et à la piste d'atterrissage aux abords du village. Tous ces milieux se caractérisent par l'absence d'arbres et au contraire la présence de végétation basse, principalement de graminées.

11. Pour une étude ethnobotanique de la diversité et l'usage des produits forestiers non ligneux, voir González Pérez 2011.

12. «Todo ambiente é ambiente de um dado organismo (ou espécie), para quem, ou de quem, o ambiente é ambiente ». 
13. Ces zones comportent des arbres, des arbustes, des plantes médicinales, des ruisseaux, et surtout des chemins, ceux tracés par les humains et ceux tracés par les animaux, qui ne doivent pas se confondre pour éviter que les humains ne laissent leur odeur sur les sentiers des animaux, ce qui aurait pour conséquence de les faire fuir.

14. SPI - Serviço de Proteçãoaos Índios (Service de protection aux Indiens), Institution précédant l'actuelle FUNAI - Fundação Nacional do Índio (Fondation nationale de l'Indien) fondée en 1967 qui dépend du Ministère de la Justice et de la Sécurité Publique brésilien.

15. Le contact s'est réalisé par des agents avec l'aide de quelques Kayapó également locuteurs de la langue Mẽbêngôkre, pour faciliter le contact. Les nouvelles techniques de pêche et la navigation ont été transmises par les agents du SPI (puis FUNAI) qui ont vécu au sein de la communauté jusqu'en 2009.

16. À propos de la mobilité Mẽbêngôkre voir Cohn (2005), Fisher (1991, 2000), Verswijver (1992) et Vidal (1977). Et pour un dialogue intéressant avec les Awá-Guajá, voir Garcia $(2010,2012)$.

17. Il s'agit du relevé des parcours que j'ai moi-même réalisés avec les membres de différentes unités résidentielles du village. Ces tracés ne correspondent donc pas à l'ensemble des chemins parcourus par toutes les familles et ne distinguent pas non plus les parcours en fonction de la saison.

18. En raison de mon sexe, et du caractère souvent individuel de la chasse qui, de surcroît, pouvait avoir lieu la nuit, il m'était plus difficile d'accompagner cette activité et d'en tirer un tracé.

19. Voir le plan de gestion territoriale et environnementale en Terre Indigène Trincheira Bacaja Aben Kaben Marimei (2018)

20. Proposition d'amendement de la Constitution, transférant au Congrès National la compétence exclusive pour l'approbation de démarcation des terres traditionnellement occupées par des groupes indigènes et la ratification des démarcations déjà homologuées, établissant ainsi un système où les critères et les procédures de démarcation seront réglementés par la loi.

http://www.camara.gov.br/proposicoesWeb/fichadetramitacao?idProposicao=14562

21. Ma traduction est issue d'une énonciation en portugais : « Se a gente não cuidar da terra, vão querer fazer alguma coisa mais rápido para sair lei para destruir nossa terra. A gente tem que tá fazendo, vigiando, cuidando para os que estão nascendo. Hoje a gente está aqui, mas amanhã a gente não está. A gente tem que preparar a terra para os que vêm nascendo »

22. Le danger des cartes a également été soulevé par les Mẽbêngôkre Kayapó dans l'article de Robert et Laques (2003) concernant un projet d'ethnocartographie réalisé auprès des Mẽbêngôkre Kayapó.

23. Pour une approche comparative de l'appropriation des nouveaux outils de représentation cartographique et de la codification de la territorialité en territoire chez les Mẽbêngôkre Kayapó, voir Robert 2004 et Robert \& Laques 2003

24. Pour une analyse critique de la prise en compte des savoirs xikrin dans l'étude des impacts socio-environnementaux, voir Mantovanelli (2016). Une comparaison de nos observations respectives permettrait d'enrichir la réflexion sur les (non-)rencontres entre différents régimes de savoir en contexte socio-environnemental conflictuel. 


\section{RÉSUMÉS}

Dans cet $\operatorname{article}^{1}$ je propose de mettre en évidence les mécanismes de transcription et de traduction d'un savoir territorial incorporé en mots et en symboles projetés sur des cartes et les nouveaux discours associés. Dans un premier temps, une analyse de la manière dont les Xikrin de la Terre Indigène Trincheira Bacajá (Pará, Brésil) entrent en relation avec leur monde forestier environnant, me permettra d'avancer que territorialité et apprentissage vont de pair avec la production de la socialité qui se concrétise dans le déplacement à travers la forêt et l'appropriation d'essences et d'espaces. Puis, dans un deuxième temps, je m'attacherai à montrer qu'un nouveau savoir, toujours ouvert, se créé à partir de la codification et de la transformation de la forêt en carte. Enfin, je montrerai comment sur la base de cette transformation, les processus relationnel et affectif qui définissent la territorialité, donnent lieu à une nouvelle conception du territoire, en tant qu'objet politique.

In this article I propose to highlight the mechanisms of transcription and translation of a territorial knowledge incorporated into words and symbols projected on maps and associated new discourses. As a first step, an analysis of how the Xikrin of the Trincheira Bacajá Indigenous Land (Pará, Brazil) relate to their surrounding forest world will allow me to argue that territoriality and learning go hand in hand with the production of sociality that is concretized in the displacement through the forest and the appropriation of species and spaces. Then, in a second step, I will try to show that a new knowledge, always open, is created from the codification and transformation of the forest into a map. Finally, I will show how, on the basis of this transformation, the relational and affective processes that define territoriality give rise to a new conception of territory as a political object.

\section{INDEX}

Keywords : Mẽbêngôkre-Xikrin, forest, territoriality, learning, innovation, mapping

Mots-clés : Mẽbêngôkre-Xikrin, forêt, territorialité, apprentissage, innovation, cartographie

\section{AUTEUR}

\section{STÉPHANIE TSELOUIKO}

Chercheure associée au Laboratoire d'anthropologie sociale, Collège de France, EHESS, CNRS, Université de recherche Paris Sciences et Lettres 